\title{
FLORA DEL CENTRO DEL ESTADO DE CHIHUAHUA, MÉXICO
}

\author{
Eduardo Estrada-CAstillón ${ }^{1}$ y José Ángel Villarreal-Quintanilla² \\ ${ }^{1}$ Universidad Autónoma de Nuevo León, Facultad de Ciencias Forestales, Apdo. \\ postal 41, 67700 Linares, Nuevo León, México. \\ ${ }^{2}$ Universidad Autónoma Agraria Antonio Narro, Departamento de Botánica, \\ Colonia Buenavista, 25315 Saltillo, Coahuila, México. \\ aeduardoestradac@prodigy.net.mx
}

\section{RESUMEN}

Se estudió la flora de las serranías y planicies de la porción central del estado de Chihuahua. Se realizaron 135 salidas de campo en un periodo de seis años, en los que se colectaron aproximadamente 6500 ejemplares botánicos, se recopiló la información bibliográfica sobre el tema y se revisó el material botánico de la región de estudio depositado en los herbarios ANSM, CFNL y TEX-LL. Se registró un total de 112 familias, 493 géneros, 1322 especies y 232 categorías infraespecíficas de plantas vasculares. Del total de familias, 87 corresponden a dicotiledóneas, 15 a monocotiledóneas, 7 a helechos y afines, y 3 a coníferas y afines. Las familias con mayor número de géneros y especies respectivamente son: Asteraceae $(86,235)$, Poaceae $(50,163)$, Leguminosae $(45,137)$, Brassicaceae $(16,25)$, Malvaceae (12, 29), Scrophulariaceae (11, 29), Cactaceae (10, 30), Verbenaceae (10, 24), Nyctaginaceae $(7,21)$ y Amaranthaceae $(7,18)$. Los géneros con mayor número de especies son Muhlenbergia (37), Dalea (22), Euphorbia (21), Cheilanthes (19), Brickellia (17), Salvia (15), Cyperus (14), Quercus (13), Solanum (12), Eragrostis (12), Bouteloua (12), Erigeron (12), Astragalus (11), Ipomoea (11), Plantago (10), Acacia (10), Machaeranthera (9), Stevia (9), Opuntia (9), Aristida (9), Asclepias (9), Phaseolus (9), Oenothera (9), Viguiera (9) y Notholaena (9). El presente estudio es una contribución hacia un mejor conocimiento de la diversidad vegetal que existe en las regiones áridas del norte de México.

Palabras clave: Chihuahua, flora, México.

\section{ABSTRACT}

In the central area of the State of Chihuahua, 135 field trips for collecting almost 6500 plant specimens were made in six years. A herbaria and bibliographic review and a study 
of the flora of the plains and low hills was also carried out. We recorded 112 families, 493 genera, 1322 species, and 232 infraspecific taxa of vascular plants. Of the total families, 87 were dicotyledons, 15 monocotyledons, 7 ferns and allies, and 3 conifers and allies. The families with highest number of genera and species were: Asteraceae (86, 235), Poaceae (50, 163), Leguminosae (45, 137), Brassicaceae (16, 25), Malvaceae (12, 29), Scrophulariaceae $(11,29)$, Cactaceae $(10,30)$, Verbenaceae $(10,24)$, Nyctaginaceae $(7,21)$ and Amaranthaceae $(7,18)$. The genera with highest number of species were: Muhlenbergia (37), Dalea (22), Euphorbia (21), Cheilanthes (19), Brickellia (17), Salvia (15), Cyperus (14), Quercus (13), Solanum (12), Eragrostis (12), Bouteloua (12), Erigeron (12), Astragalus (11), Ipomoea (11), Plantago (10), Acacia (10), Machaeranthera (9), Stevia (9), Opuntia (9), Aristida (9), Asclepias (9), Phaseolus (9), Oenothera (9), Viguiera (9) and Notholaena (9).The present study is a contribution to the knowledge of plant diversity in the arid lands of northern Mexico.

Key words: Chihuahua, flora, Mexico.

\section{INTRODUCCIÓN}

Chihuahua es el estado más grande de la República Mexicana; abarca una superficie de $247,087 \mathrm{~km}^{2}$, de la cual casi $70 \%$ corresponde a zonas áridas y semiáridas. Los contrastes de relieve, suelo y clima hacen patente una conformación heterogénea de su cubierta vegetal, Shreve (1939) reconoce cuatro tipos de vegetación dominantes en el estado: desierto, bosque de encino y pastizales, bosque de pino y la vegetación de barranca; LeSueur (1945) describe para la mitad norte de la entidad siete: bosque montano, bosque de Quercus grisea, bosque de $Q$. santaclarensis, bosques mixtos de encino, bosque espinoso, pastizal de Bouteloua gracilis y matorral desértico; Rzedowski (1978) ubica cuatro tipos de vegetación para el estado: matorral xerófilo, pastizal, bosque de coníferas y encinos y bosque tropical caducifolio.

Con respecto a la flora, en la actualidad no se cuenta con un listado completo, aunque se han llevado a cabo estudios en algunas familias tales como helechos y afines (Knobloch y Correll, 1962), gramíneas (Valdés et al., 1975; Lebgue y Valerio, 1991; Beettle, 1983, 1987, 1991, 1995) y leguminosas (Estrada y Martínez, 2000). Para el área del centro de Chihuahua, existen referencias aisladas para algunos grupos de plantas como Quercus (Muller, 1979; Nixon, 1998), musgos (Delgadillo, 1998), helechos (Riba, 1998), Acanthaceae (Daniel, 1998), Agavaceae (Gentry, 1982), Asteraceae (Turner y Nesom, 1998) y Nolinaceae (García-Mendoza y Galván, 1995), mientras que para la porción sur se han realizado escasos estudios de flora (Spellenberg et al., 1996; Estrada et al., 1997; Laferriere, 1994). 
La presente contribución se basa en las colectas de los autores en la región del centro del estado de Chihuahua durante seis años de exploraciones intensivas en todas las comunidades vegetales, así como en la recopilación bibliográfica y la consulta del material depositado en los herbarios de la Universidad Autónoma Agraria Antonio Narro (ANSM), Facultad de Ciencias Forestales de la Uiversidad Autónoma de Nuevo León (CFNL) y de la Universidad de Texas en Austin (TEX-LL). Los ejemplares se identificaron por los autores, cuando hubo dudas al respecto se enviaron a especialistas para su correcta determinación. Para el estudio realizado se tomó en cuenta asimismo la información acumulada en la flora del Desierto Chihuahuense de James S. Henrickson y Marshall C. Johnston (1997), obra aún inédita, con gran valor científico para el conocimiento de la flora mexicana de zonas áridas y semiáridas del norte y centro de nuestro país.

El objetivo del presente trabajo es enlistar y cuantificar la diversidad de especies de plantas vasculares existentes en la región semiárida del centro chihuahuense.

\section{DESCRIPCIÓN DEL ÁREA DE ESTUDIO}

La región central del estado de Chihuahua se caracteriza por sus extensas planicies y lomeríos bajos en sus sectores oeste, este y sur, donde predominan el pastizal y el matorral, mientras que en la porción norte se encuentra una cadena de montañas conocidas como sierra El Nido, donde queda incluido el Parque Nacional Cumbres de Majalca. Esta parte más elevada, con climas más frescos, alberga una vegetación y flora diferente con respecto a las planicies y lomeríos, pues en ella dominan los encinares y pinares.

Fisiografía. La región del centro del estado de Chihuahua se encuentra dentro de dos provincias fisiográficas: Sierra y Llanuras Tarahumaras y Sierras y Llanuras del Norte; esta última engloba tres subprovincias: Bolsón de Mapimí, Llanuras y Sierras Volcánicas y Sierras Plegadas del Norte (Anónimo, 1987), mismas que forman parte de la región florística xerofítica mexicana (Rzedowski, 1978). El área de estudio se localiza entre $\operatorname{los} 28^{\circ} 00^{\prime}-30^{\circ} 07^{\prime} \mathrm{N}$ y $104^{\circ} 25^{\prime}-106^{\circ} 35^{\prime} \mathrm{W}$, en altitudes de 1450-2300 m; incluye una superficie aproximada de 21,700 km², abarcando territorios de once municipios: General Trías, Chihuahua, Coyame, Aldama, Aquiles Serdán, Julimes, Meoqui, Delicias, Ahumada, Camargo y Riva Palacio (Fig. 1). El relieve es heterogéneo, la mayor parte de las extensas planicies y lomeríos bajos están constituidos por depósitos sedimentarios y vulcanosedimentarios del Cuaternario. 

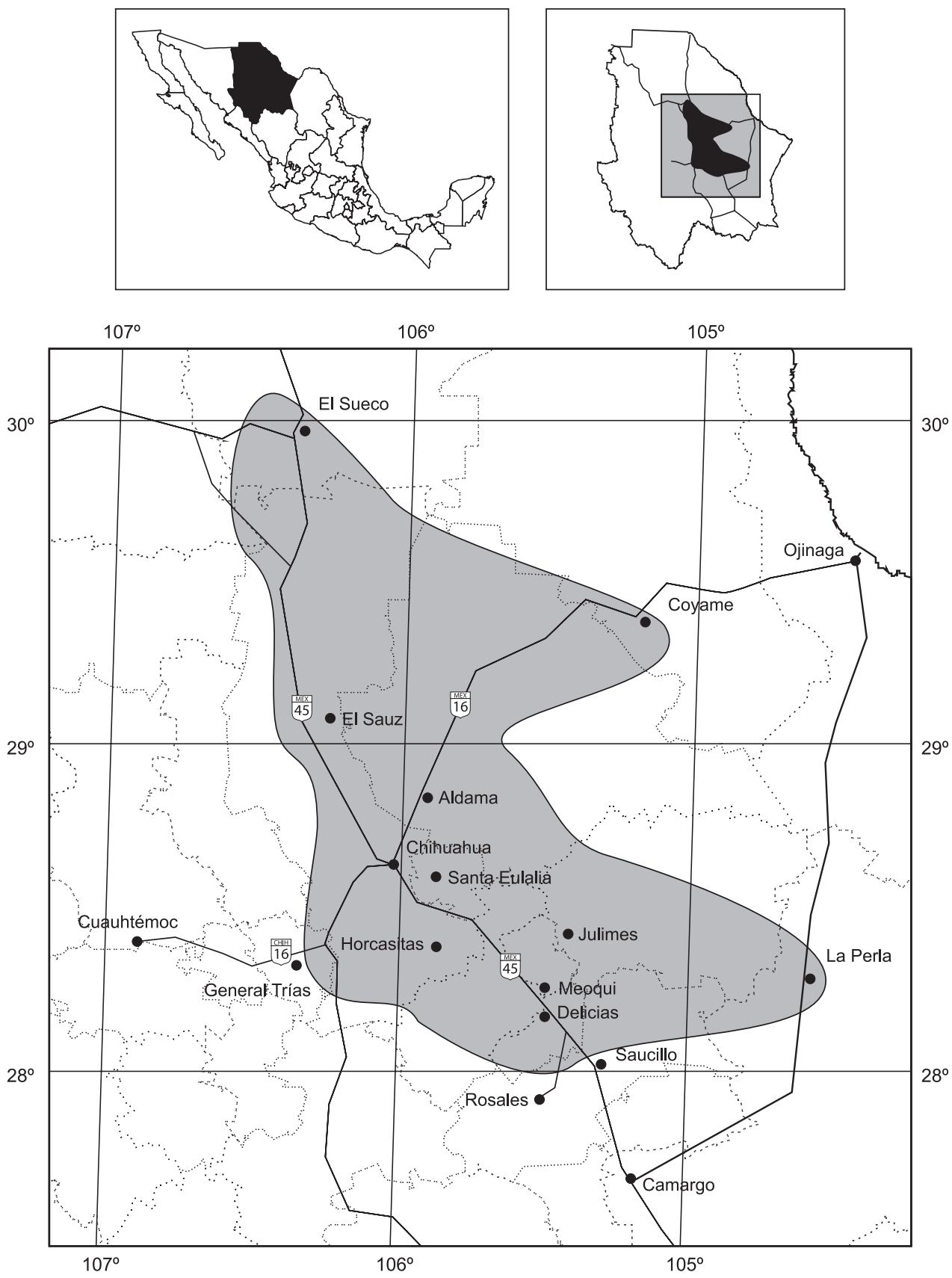

Fig. 1. Área de estudio, incluye superficies de once muncipios: General Trías, Chihuahua, Coyame, Aldama, Aquiles Serdán, Julimes, Meoqui, Delicias, Ahumada, Camargo y Riva Palacio. 
Las montañas más altas se localizan al noroeste de ciudad Chihuahua, comprenden el Parque Nacional Cumbres de Majalca.

Geología. Las serranías presentan regularmente una dirección noroeste-sureste, compuesta en el mayor de los casos por rocas ígneas extrusivas ácidas del Terciario y rocas sedimentarias del Cretácico Superior de tipo arenisca y caliza. Áreas localizadas al noreste de Ciudad Chihuahua presentan rocas del Paleozoico Superior de tipo lutitas, areniscas y calizas y del Jurásico de tipo lutitas y areniscas (Anónimo, 1981a).

Clima. Los climas predominantes en la porción central del estado corresponden a los tipos $\mathrm{BS}_{0} \mathrm{kw}$ y $\mathrm{BS}_{0} \mathrm{hw}$, ambos de carácter estepario, con 300-400 mm de precipitación anual y una temperatura media anual entre 16 y $18^{\circ} \mathrm{C}$, así como BWkw y BWhw, desértico, con 200-300 mm de precipitación anual y una temperatura media anual de $18-20{ }^{\circ} \mathrm{C}$ (Anónimo, 1981b), ambos son secos, con lluvias en verano, templados (k) y semicálidos (h) (García, 1973).

En el área de estudio, el tipo BS predomina desde Ciudad Chihuahua hasta El Sueco (García, 1973; Anónimo, 1981b), con variantes en temperatura y precipitación. En Ciudad Chihuahua se presenta el tipo BS $\mathrm{BW}_{0}(\mathrm{w})(\mathrm{e}$ ), con un porcentaje de precipitación invernal de 3.7. La temperatura media es de $18.4{ }^{\circ} \mathrm{C}$, los meses más calientes son mayo, junio, julio, agosto y septiembre con 24, 26.8, 25.2, 24.3 y 22.3 ${ }^{\circ} \mathrm{C}$ cada uno. La precipitación anual en promedio es de $347 \mathrm{~mm}$, los meses más lluviosos son junio, julio, agosto y septiembre. La estación El Sueco (2954' N-106 $23^{\circ}$ W) presenta el tipo $\mathrm{BS}_{1} \mathrm{kw}\left(\mathrm{e}^{\prime}\right)$, con un porcentaje de precipitación invernal de 7.8. La temperatura media anual es de $14.9^{\circ} \mathrm{C}$, los meses más calientes son junio, julio, agosto y septiembre, con 23.6, 24, 22.8 y $20.2^{\circ} \mathrm{C}$ cada uno. La precipitación anual es en promedio de $343 \mathrm{~mm}$, los meses más lluviosos son julio, agosto y septiembre con 83, 83.3 y 70.7 mm cada uno.

Se carece de registros del clima de la región de Cumbres de Majalca, pero en función de su vegetación cabe deducir que al menos en alguas porciones del área predominan algunas variantes del tipo $\mathrm{Cw}$.

Los climas BW se localizan al noreste de la estación El Sueco, en porciones del municipio de Aldama y al este de Ciudad Delicias (García, 1973; Anónimo, 1981b). Se presentan dos variantes principales, en Ciudad Delicias, Meoqui y partes de Julimes predomina el BWhw(e'), con un porcentaje de precipitación invernal de 5.1. La temperatura media anual es de $18.8^{\circ} \mathrm{C}$, los meses más calientes son mayo, junio, julio, agosto y septiembre con $24,27,26.2,25.3$ y $23{ }^{\circ} \mathrm{C}$ cada uno. La precipitación anual en promedio es de 281 mm, los meses más lluviosos son junio, julio, 
agosto y septiembre, con 29.4, 62.4, 66.9, y $55.3 \mathrm{~mm}$ respectivamente. En Ciudad Aldama (30 km al noreste de Ciudad Chihuahua) se presenta el BWhw(w)(e')w" con un porcentaje de precipitación invernal de 4.2. La temperatura media anual es de $19.3^{\circ} \mathrm{C}$, los meses más calientes son mayo, junio, julio y agosto con 23.6, 26.7, 26.4, y $25.2{ }^{\circ} \mathrm{C}$ cada uno. La precitación anual en promedio es de $330 \mathrm{~mm}$, los meses más lluviosos son julio, agosto y septiembre con 81.3 , 72.2 y 76.8 mm cada uno.

Vegetación. En el área de estudio se presentan tres tipos de vegetación principales: matorral xerófilo, pastizal, bosque de encino-pino (Rzedowski, 1978). A su vez, dentro de éstos cabe reconocer: pastizal mediano abierto, pastizal mediano arborescente, pastizal amacollado abierto, pastizal halófilo, matorral inerme parvifolio, matorral rosetófilo y bosque esclero-aciculifolio (Anónimo, 1978).

Pastizal mediano abierto. Se encuentra constituido por gramíneas de porte mediano (0.5-1 m) con un claro predominio de "navajitas" (Bouteloua spp.), entre las cuales Bouteloua gracilis es la más común, además de Aristida spp., Bouteloua breviseta, B. chondrosioides, B. curtipendula, B. eriopoda, B. hirsuta, Digitaria californica, Enneapogon desvauxii, Eragrostis intermedia, Hilaria mutica, Leptochloa dubia, Lycurus phleoides y Panicum obtusum. El sobrepastoreo y la perturbación actual han erradicado casi en su totalidad a esta comunidad, de tal modo que son pocas las áreas donde hoy puede observarse en su forma prístina. Muchos sitios de planicie, en los cuales existía tal vegetación al norte de Ciudad Chihuahua y en partes de los municipios de General Trías, Aquiles Serdán, Chihuahua, Aldama, Coyame y Ahumada, han sido invadidos por especies arbustivas indeseables, como Prosopis glandulosa var. torreyana y Mimosa aculeaticarpa var. biuncifera.

Pastizal mediano arborescente. Representa una transición entre el pastizal y el bosque de encino; su distribución es frecuente en las faldas de la Sierra Madre Occidental y en sierras aisladas al oeste y norte de Ciudad Chihuahua. Ocupa parte de los municipios de Chihuahua y General Trías. En esta comunidad se diferencian dos estratos de vegetación principal, uno de gramíneas de porte mediano (50-75 cm) y otro de especies arbóreas bajas, con hojas escamosas, aciculares o esclerotizadas. Las plantas herbáceas dominantes son Aristida spp., Bouteloua gracilis, $B$. hirsuta, B. chondrosioides, Bouteloua dactyloides, Heteropogon contortus, Lycurus phleoides, Muhlenbergia minutissima, Panicum hallii y Setaria macrostachya. Los árboles característicos son Juniperus monosperma, Pinus cembroides, Quercus chihuahuensis, Q. emoryi y Q. grisea. 
Pastizal amacollado abierto. Esta comunidad está conformada por gramíneas amacolladas perennes y cubre gran parte de serranías aisladas. En la zona central cubre parte de los municipios de Ahumada, Chihuahua, Aldama, Coyame, Julimes, Sacramento y Santa Eulalia. Los pastos dominantes son del género Bouteloua, destacando B. curtipendula, B. eriopoda, B. hirsuta y B. trifida, asociadas con Bothriochloa barbinodis, Enneapogon desvauxii, Heteropogon contortus, Lycurus phleoides, Muhlenbergia emersleyi, Trachypogon spicatus; las plantas arbustivas frecuentes son Mimosa dysocarpa, Mimosa pringlei, Rhus trilobata, Rhus virens y Salvia spp., presentando diversas modalidades de asociación y fisonomía.

Pastizal halófilo. Conformado por gramíneas perennes que constituyen una carpeta uniforme, con predominio de pocas especies resistentes y adaptadas a suelos salinos, alcalinos, mal drenaje y escurrimiento lento. Se distribuye regularmente en las partes más bajas de planicies y cuencas cerradas húmedas. Ocupa parte de los municipios de Aldama, Chihuahua y Ahumada. Los suelos alcanzan $50 \mathrm{~cm}$ o más de profundidad, de textura arcillo-limosa, pH entre 6.7 y 10.3. En este pastizal destaca la presencia de Sporobolus airoides, Dasyochloa pulchella, Hilaria belangeri, Distichlis spicata var. stricta, Hilaria mutica, Eragrostis obtusiflora, Muhlenbergia repens, Panicum obtusum, Scleropogon brevifolius y Sporobolus pulvinatus, además de otros elementos herbáceos y arbustivos como Atriplex canescens, Condalia ericoides, Flourensia cernua, Gutierrezia microcephala, Koeberlinia spinosa, Larrea tridentata y Salsola tragus.

Matorral inerme parvifolio. Lo constituyen plantas arbustivas de porte bajo, menor de $2 \mathrm{~m}$, con predominio de elementos inermes. Se encuentra ampliamente disperso en la porción oriental y norte del estado; cubre porciones de los municipios de Chihuahua, Aldama, Coyame, Julimes, Santa Eulalia, Delicias, Meoqui y Ahumada. Los taxa predominantes son Acacia neovernicosa, Aloysia gratissima, A. wrightii, Ambrosia spp., Celtis pallida, Condalia ericoides, Flourensia cernua, Fouquieria splendens, Jatropha dioica, Larrea tridentata, Opuntia spp., Parthenium argentatum, $P$. incanum y Rhus microphylla. En algunas áreas de este tipo de vegetación es manifiesta la presencia de manchones con predominio de especies espinosas, a mencionar Acacia constricta, A. neovernicosa, Koeberlinia spinosa y Prosopis glandulosa var. torreyana. Las gramíneas más comunes son Bouteloua spp., Digitaria californica, Leptochloa dubia, Lycurus phleoides y Setaria macrostachya. 
Matorral rosetófilo. Se caracteriza por el predominio de elementos con hojas crasas, dispuestas en roseta y culminando en espina o de otras con presencia de espinas en el margen de la hoja y tallo y hojas. Esta comunidad vegetal se localiza principalmente en las serranías bajas de la porción oriental del estado. En el área de estudio se localiza en parte de los municipios de Aldama y Coyame, donde son preponderantes Agave lecheguilla, Dasylirion spp., Nolina texana y Yucca spp., asociadas a Acacia greggii, Berberis trifoliolata, Celtis pallida, Ephedra trifurca, Euphorbia antisyphilitica, Fouquieria splendens, Jatropha dioica y gramíneas como Aristida spp., Bothriochloa barbinodis, Bouteloua curtipendula, B. gracilis, B. hirsuta, Enneapogon desvauxii y Heteropogon contortus.

Bosque esclero-aciculifolio. Lo constituye la asociación de dos géneros de plantas, Quercus y Pinus. Se localiza en las partes medianas y más altas de las montañas. En el área de estudio comprende parte del municipio de Chihuahua (Parque Nacional Cumbres de Majalca, Sierra El Nido, Cerro La Campana). Las dominantes son Cupressus arizonica, Juniperus monosperma, J. deppeana, Pinus cembroides, P. engelmannii, Quercus arizonica, Q. chihuahuensis, Q. emoryi, Q. hypoleucoides y las gramíneas asociadas son Aristida spp., Bouteloua gracilis, B. curtipendula, $B$. hirsuta, Bromus carinatus, Elyonurus barbiculmis, Eragrostis intermedia, Lycurus phleoides, Muhlenbergia emersleyi, M. monticola, M. rigens y Panicum bulbosum, entre las más frecuentes.

\section{MÉTODOS}

Durante los años 1994-2001 se realizaron 135 salidas de campo para colecta de ejemplares botánicos. Las obtención de especímenes se realizó en diversas estaciones del año con el fin de registrar los diferentes estados fenológicos de las especies en todos los tipos de vegetación presentes en el centro del estado. En cada sitio de se tomaron datos sobre la comunidad vegetal dominante, altitud, coordenadas geográficas, poblados cercanos, exposición, pendiente. La identificación de especimenes fue realizada por los autores en los herbarios ANSM y CFNL. Aquellos de difícil identificación fueron enviados a los especialistas para su corroboración. La base de los especimenes se encuentra en los herbarios ANSM y CFNL, otra parte lo está en BRIT, CIIDIR, MEXU, TEX/LL, UAT y US. Se consultó el estudio de Henrickson y Johnston (1997) para incluir aquellos taxa que no fueron registrados en las colectas. En el listado florístico se sigue el sistema de clasificación de Cronquist et al. 
(1966) para nombres de categorias de división y clase, Cronquist (1981) para magnoliofitas y Crabbe et al. (1975) para helechos y afines a éstos. Las categorías de familia, género y especie en cada grupo, se presentan en secuencia alfabética.

\section{RESULTADOS}

De acuerdo con este inventario, la flora vascular del centro del estado de Chihuahua está constituida por 112 familias, 493 géneros, 1322 especies y 232 categorías infraespecíficos de plantas vasculares (Apéndice). El cuadro 1 muestra los números totales correspondientes a dicotiledóneas, monocotiledóneas, helechos y afines, y a coníferas y afines. Las familias con más géneros y especies respectivamente son: Asteraceae $(86,235)$, Poaceae $(50,163)$, Leguminosae $(45,137)$, Brassicaceae (16, 25), Malvaceae (12, 29), Scrophulariaceae (11, 29), Cactaceae (10, 30), Verbenaceae $(10,24)$, Nyctaginaceae $(7,21)$ y Amaranthaceae $(7,18)$. Los géneros más diversificados son Muhlenbergia (37), Dalea (22), Euphorbia (21), Cheilanthes (19), Brickellia (17), Salvia (15), Cyperus (14), Quercus (13), Solanum (12), Eragrostis (12), Bouteloua (12), Erigeron (12), Astragalus (11), Ipomoea (11), Plantago (10), Acacia (10), Machaeranthera (9), Stevia (9), Opuntia (9), Aristida (9), Asclepias (9), Phaseolus (9), Oenothera (9), Viguiera (9) y Notholaena (9).

Cuadro 1. Diversidad de familias, géneros y especies de la flora del centro del estado de Chihuahua.

\begin{tabular}{lcrcc}
\hline & Familias & Géneros & Especies & $\begin{array}{c}\text { Categorías } \\
\text { infraespecíficas }\end{array}$ \\
\hline Pteridophyta & 7 & 16 & 58 & 6 \\
Pinophyta & 3 & 4 & 13 & 1 \\
Magnoliophyta & & & & \\
$\quad$ Magnoliopsida & 87 & 394 & 1018 & 193 \\
$\quad$ Liliopsida & 15 & 79 & 233 & 32 \\
TOTAL & 112 & 493 & 1322 & 232 \\
\hline
\end{tabular}

De acuerdo con Rzedowski (1998), la flora fanerogámica de México está constituida por 220 familias, 2410 géneros y 22000 especies, con fines de comparación 
con el área de estudio, el centro de Chihuahua posee 50.9\% de familias, 20.45\% de géneros y 6\% de las especies presentes en México. Asimismo, nuestros resultados referentes a familias, géneros y especies concuerdan con los mencionados por Rzedowski (1998) al destacar que las familias Asteraceae, Leguminosae y Poaceae son los grupos de plantas mejor representados en México.

La flora vascular del área está constituida por ocho formas biológicas principales: hierbas, arbustos inermes, árboles, enredaderas, plantas crasicaules, arbustos espinosos, arbustos rosetófilos y parásitos. Las especies herbáceas predominan sobre el resto con 1252 taxa (86\%), seguidas por arbustivas inermes (81) (5.5\%), arbustivas espinosas (81) (5.5\%) y árboles (44) (3\%).

Los elementos arbóreos están representados por las familias Cupressaceae (7 especies), Pinaceae (3), Fagaceae (11), Juglandaceae (2), Leguminosae (7), Oleaceae (1), Rosaceae (3), Rubiaceae (1), Salicaceae (5), Sapindaceae (1), Tamaricaceae (2) y Ulmaceae (1).

En conjunto, las plantas arbustivas engloban 161 especies (11.05\%), de las que varias son dominantes en las comunidades de matorral en planicies y lomeríos del área de estudio; destacan por su densidad y cobertura Larrea tridentata, Acacia neovernicosa, Flourensia cernua, Parthenium confertum y Parthenium incanum. Estos matorrales son afines en composición y estructura a los respectivos de la zona árida del estado de Durango, reportados por González et al. (1993).

Las especies arbóreas concentran su mayor diversidad y distribución en las partes montañosas más altas, especialmente en el Parque Nacional Cumbres de Majalca, donde Pinus leiophylla var. chihuahuana, P. cembroides, P. engelmannii, Cupressus arizonica, Fraxinus velutina, Quercus hypoleucoides, Q. rugosa y Q. grisea brindan la fisonomía característica de bosque al área (Estrada et al., 2003).

La flora del centro del estado de Chihuahua, comparada con la de la Casacada de Basaseachic (Spellenberg et al., 1996) y la de la Laguna de Babícora (Estrada et al., 1997), permite conocer su riqueza (Cuadro 2) y afinidad. El centro de

Cuadro 2. Comparación de diversidad de floras en tres áreas del estado Chihuahua.

\begin{tabular}{lcccc}
\hline & Familias & Géneros & Especies & Superficie $\left(\mathrm{km}^{2}\right)$ \\
\hline Centro de Chihuahua & 112 & 493 & 1322 & 21700 \\
Cascada de Basaseachi & 117 & 414 & 823 & 60 \\
Laguna de Babícora & 69 & 247 & 489 & 1882 \\
\hline
\end{tabular}


Chihuahua tiene una similitud de 79\% de familias, 40\% de géneros y sólo 1\% de las especies con respecto a la Cascada de Basaseachi, mientras que con la Laguna de Babícora la similitud es de $99 \%$ para familias, $60 \%$ con géneros y $26.9 \%$ con especies.

\section{AGRADECIMIENTOS}

Se agradece la colaboración de las siguientes personas. A Miguel Ángel González Botello por la elaboración del mapa. A Thomas Wendt por permitirnos la consulta de información del herbario TEX/LL. A Guy L. Nesom y B. L. Turner por la identificación de material crítico de la familia Asteraceae. A Jesús Valdés Reyna y Kelly W. Alrred por la ayuda en la determinación y corroboración de especímenes de la familia Poaceae. A Socorro González Elizondo por su ayuda en la identificación y corroboración de ejemplares de las familias Cyperaceae y Juncaceae. A Richard Spellenberg por la revisión del escrito e información de especies registradas en el área de estudio almacendas en el herbario NMC. A Rosa María Murillo por sus valiosas aportaciones y revisión crítica del manuscrito y a los revisores anónimos de la revista. A Guadalupe Martínez Ávalos y Miguel Ángel González Botello por su valiosa cooperación en la identificación y corroboración de materiales de la familia Cactaceae. A James S. Henrickson y Marshall C. Johnston por permitirnos revisar su valioso trabajo sobre la Flora de la Región del Desierto Chihuahuense. A Carmen Yen Méndez, Claudia Castillo Moreno y María Alfaro Martínez por su trascendente ayuda en la colecta de material botánico. A Enrique Jurado por la revisión del abstract.

\section{LITERATURA CITADA}

Anónimo. 1978. Guías para la determinación de condición. Comisión Técnico Consultiva para la Determinación de los Coeficientes de Agostadero. Secretaría de Agricultura y Recursos Hidráulicos. México, D.F. 78 pp.

Anónimo. 1981a. Chihuahua. Carta geológica, escala 1: 1000 000. Instituto Nacional de Estadística Geografía e Informática, Secretaría de Programación y Presupuesto. México, D.F.

Anónimo. 1981b. Chihuahua. Carta de climas, escala 1: 1000 000. Instituto Nacional de Estadística Geografía e Informática, Secretaría de Programación y Presupuesto. México, D.F. 
Anónimo. 1987. Chihuahua, Mapa 1.2, Fisiografía, escala 1:2000 000. Instituto Nacional de Estadística Geografía e Informática, Secretaría de Programación y Presupuesto. México, D.F.

Beetle, A. A. 1983. Las gramíneas de México. Tomo I. Secretaría de Agricultura y Recursos Hidráulicos. México D.F. 260 pp.

Beetle, A. A. 1987. Las gramíneas de México. Tomo II. Secretaría de Agricultura y Recursos Hidráulicos. México D.F. 344 pp.

Beetle, A. A. 1991. Las gramíneas de México. Tomo III. Secretaría de Agricultura y Recursos Hidráulicos. México D.F. 322 pp.

Beetle, A. A. 1995. Las gramíneas de México. Tomo IV. Secretaría de Agricultura, Ganadería y Desarrollo Rural. México D.F. 342 pp.

Crabbe, J. A., A. C. Jermy y J. T. Mickel. 1975. A new generic sequence for the pteridophyte herbarium. Fern Gaz. 11(2/3): 141-162.

Cronquist, A. 1981. An integrated system of clasification of flowering plants. Columbia University Press, New York.

Cronquist, A., A. Takhtajan y W. Zimmermann. 1966. On the higher taxa of Embryophyta. Taxon 14(4): 129-134.

Daniel, T. F. 1998. Acanthaceae de México: diversidad y distribución In: Ramamoorthy, T. P. Bye, R. Lot A. y J. Fa. (eds.). Diversidad biológica de México: Orígenes y distribución. Instituto de Biología. Universidad Nacional Autónoma de México, México, D.F. pp. 527-544.

Delgadillo, C. 1998. Diversidad de la brioflora mexicana. In: Ramamoorthy, T. P., Bye, R., Lot, A. y J. Fa. (eds.). Diversidad biológica de México: Orígenes y distribución. Instituto de Biología. Universidad Nacional Autónoma de México, México, D.F. pp. 355-368.

Estrada A. E., R. Spellenberg y T. Lebgue. 1997. Flora vascular de la Laguna de Babícora, Chihuahua, México. Sida 17(4): 809-827.

Estrada A. E. y A. Martínez. 2000. Legumes from the central part of the state of Chihuahua, Mexico. Sida 19(2): 351-360.

Estrada, A. E., E. Jurado, J. J. Návar, J. Jiménez y F. Garza. 2003. Plant associations of Cumbres de Majalca National Park, Chihuahua, México. The Southw. Nat. 48(2): 177-187.

García, E. 1973. Modificaciones al sistema de clasificación climática de Koeppen. Instituto de Geografía. 2a ed. Universidad Nacional Autónoma de México. México, D.F. 217 pp.

García-Mendoza, A. y R. Galván. 1995. Riqueza de las familias Agavaceae y Nolinaceae en México. Bol. Soc. Bot. Méx. 56: 7-24.

Gentry, H. S. 1982. Agaves of continental North America. University of Arizona Press. Tucson, Arizona. 670 pp.

González E., S., M. González E. y A. Cortés-Ruiz. 1993. Vegetación de la Reserva de la Biósfera “La Michilia”, Durango, México. Acta Bot. Mex. 22: 1-104.

Henrickson, J. y M. C. Johnston. 1997 (inédito). A flora of the Chihuahuan Desert region. Los Angeles, California. 1687 pp.

Knobloch, I. W. y D. S. Correll. 1962. Fern and fern allies of Chihuahua, Mexico. Contr. Tex. Res. Found. No. 3. 198. pp. 
Laferriere, J. E. 1994. Vegetation and flora of the mountain Pima village of Nabogame, Chihuahua, Mexico. Phytologia 77(2): 102-140.

Lebgue, T. y A. Valerio. 1991. Gramíneas de Chihuahua. Universidad Autónoma de Chihuahua. Chihuahua, Chih. 301 pp.

LeSueur, H. 1945. The ecology of the vegetation of Chihuahua, Mexico, north of the parallel twenty-eight. University of Texas Publications No. 452.92 pp.

Muller, C. H. 1979. Quercus deliquescens, a new species from Chihuahua, Mexico. Phytologia 42(4): 289-291.

Nixon, K. C. 1998. El género Quercus en México. In: Ramamoorthy, T. P., R. Bye, A. Lot. y J. Fa (eds.). Diversidad biológica de México: orígenes y distribución. Instituto de Biología, Universidad Nacional Autónoma de México. México, D.F. pp. 435-447.

Riba, R. 1998. Pteridofitas mexicanas: Distribución y endemismo. In: Ramamoorthy, T. P., Bye, R., Lot, A. y J. Fa (eds.): Diversidad biológica de México: orígenes y distribución. Instituto de Biología, Universidad Nacional Autónoma de México, México, D.F. pp. 369-384.

Rzedowski, J. 1978. Vegetación de México. Ed. Limusa. México, D.F. pp. 97-110.

Rzedowski, J. 1998. Diversidad y orígenes de la flora fanerogámica de México. In: Ramamoorthy, T. P., R. Bye, A. Lot y J. Fa (eds.). Diversidad biológica de México: Orígenes y distribución. Instituto de Biología, Universidad Nacional Autónoma de México. México, D.F. pp. 129-145.

Shreve, F. 1939. Observations on the vegetation of Chihuahua. Madroño 5: 1-48.

Spellenberg, R. T. Lebgue y R. Corral. 1996. Listados florísticos de México. XIII. A specimen-based, annotated checklist of the vascular plants of Parque Nacional "Cascada de Basaseachi" and adjacent areas, Chihuahua, México. Instituto de Biología, Universidad Nacional Autónoma de México. México, D.F. 72 pp.

Turner, B. L. y G. L. Nesom. 1998. Biogeografía, diversidad y situación de peligro o amenaza de Asteraceae de México. In: Ramamoorthy, T. P., R. Bye, A. Lot. y J. Fa (eds.). Diversidad biológica de México: orígenes y distribución. Instituto de Biología, Universidad Nacional Autónoma de México. México, D.F. pp. 545-561.

Valdés, J., A. A. Beetle y M. H. González. 1975. Gramíneas de Chihuahua. Pastizales 4(3): $2-60$. 


\section{APÉNDICE}

\section{Lista florística}

Los números de colecta pertenecen al primer autor. Los números sin acrónimo indican que los ejemplares están depositados en el herbario CFNL. las siglas sin paréntisis corresponden al herbario donde está depositado el ejemplar. Los acrónimos ARIZ, MEXU, NMC TEX o US, anotados entre paréntesis y seguidos por un número, corresponden al número del ejemplar de la especie en la colección científica de tales herbarios y que ha sido colectado en el centro del estado de Chihuahua. Los taxa precedidos por uno a tres asteriscos no fueron encontrados por los autores pero han sido registrados para el área de estudio por otros investigadores: los marcados con un asterisco son los referidos por Henrickson y Johnston (1997), con dos por Peterson y Annable (1991), y con tres por Lebgue y Valerio (1991).

\section{PTERIDOPHYTA}

\section{ASPLENIACEAE}

Asplenium palmeri Maxon, (TEX 237584, 237586).

Asplenium trichomanes L. ssp. trichomanes *

Phanerophlebia auriculata Underw., (TEX 243030).

Woodsia mexicana Fée, (TEX 243311).

Woodsia phillipsii Windham, (TEX 243269).

DRYOPTERIDACEAE

Dryopteris patula (Sw.) Underw., 8766 ANSM, BRIT, TEX.

EQUISETACEAE

Equisetum laevigatum A. Braun, 9694.

MARSILEACEAE

Marsilea ancylopoda A. Braun, 5595 ANSM; 8385 ANSM.

POLYPODIACEAE

Pleopeltis polylepis (Kuntze) T. Moore var. erythrolepis (Weath.) T. Wendt, 6423.

Pleopeltis riograndensis (T. Wendt) E. G. Andrews \& Windham, (TEX 232328).

Polypodium erythrolepis (Weath.) Pic. Serm., 6423. 
Polypodium thyssanolepis (A. Braun ex Klotzsch) E.G. Andrews \& Windham, 8741 TEX; 8915 TEX; (TEX 232345).

\section{PTERIDACEAE}

Adiantum capillus-veneris L., (TEX 236376).

Adiantum tricholepis Fée, (TEX 236622).

Argyrochosma formosa (Liebm.) Windham, (TEX 214687).

Argyrochosma limitanea (Maxon) Windham ssp. limitanea (TEX 269689).

Argyrochosma limitanea (Maxon) Windham ssp. mexicana (Maxon) Windham, (TEX 269699, 269701, 269702).

Argyrochosma microphylla (Mett. ex Kuhn) Windham, 7970 TEX; 8869 ANSM, BRIT, TEX, (TEX 269730, 269736, 269737, 269742).

Astrolepis cochisensis (Goodd.) D.M. Benham \& Windham, 5792 ANSM, TEX; (TEX 268228, 268230, 268252).

Astrolepis integerrima (Hook.) D.M. Benham \& Windham, (TEX 268297, 268318, 268335).

Astrolepis sinuata (Sw.) (Lag. ex Sw.) D.M. Benham \& Windham, 5454 ANSM; 5860 ANSM, TEX; 8390 ANSM, TEX; (NMC 52890; TEX 268378, 268380, 268429).

Bommeria hispida (Mett.) Underw., 4933 ANSM, TEX; 7359 ANSM, TEX; 8469 ANSM; 8789 ANSM; (TEX 236142, 236143, 236145).

Cheilanthes alabamensis (Buckley) Kuntze, (TEX 268454).

Cheilanthes allosuroides Mett., (TEX 268509).

Cheilantes arizonica (Maxon) Mickel *

Cheilanthes bonariensis (Willd.) Proctor, (NMC 52889; TEX 268571, 268573, 268576).

Cheilantes cucullans Fée *

Cheilanthes eatonii Baker, 8328 ANSM, BRIT; 8749 ANSM; (TEX 268829, 268835, 268843).

Cheilanthes feei T. Moore, (TEX 268878).

Cheilanthes farinosa (Forssk.) Kaulf., 8398 ANSM.

Cheilanthes horridula Maxon, (TEX 214692).

Cheilanthes kaulfussii Kunze, (TEX 269029, 269030).

Cheilanthes leucopoda Link, (TEX 269067, 269073).

Cheilanthes lindheimeri Hook., 7954 TEX; 8222 ANSM; 8815 ANSM; (TEX 269093, 269089, 269094, 269102).

Cheilanthes mexicana Davenp., (TEX 269156, 269157, 269164).

Cheilanthes microphylla (Sw.) Sw., (TEX 269182).

Cheilanthes myriophylla Desv., (TEX 269222).

Cheilanthes notholaenoides Maxon ex Weath. * 
Cheilanthes tomentosa Link, 8737 ANSM.

Cheilanthes villosa Maxon, (TEX 269322, 269323).

Cheilanthes wrightii Hook., (NMC 52888; TEX 269360, 269361, 269363, 269370).

Notholaena aliena Maxon *

Notholaena aschenborniana Klotzsch, 7777 ANSM, BRIT; 7947 ANSM; 7952 ANSM; 8013a ANSM; 8324 ANSM, TEX; 8754 ANSM, TEX; (TEX 269440, 269446).

Notholaena bryopoda Maxon *

Notholaena candida (M. Martens \& Galeotti) Hook. *

Notholaena grayi Davenp., (TEX 269612, 269613).

Notholaena greggii (Mett. ex Kuhn). Maxon, (TEX 214697, 269620).

Notholaena neglecta Maxon *

Notholaena schaffneri (E. Fourn.) Underw., (TEX 269715, 269726, 269717, 269727).

Notholaena standleyi Maxon, 8548 ANSM, TEX; (TEX 214698).

Pellaea cordifolia (Sessé \& Moc.) A.R. Sm., 7975 TEX; 8772 ANSM, BRIT, TEX.

Pellaea intermedia Mett. ex Kuhn, (TEX 269963).

Pellaea ternifolia (Cav.) Link var. ternifolia, 7267 TEX; 7360 ANSM, TEX; 8396 ANSM, TEX; 8673 ANSM; 8742 NMC; 8755 TEX; 8756 ANSM; (TEX 236027, 236029, 336017). Pellaea ternifolia (Cav.) Link var. wrightiana (Hook.) A.F. Tryon, 7746 TEX.

Pellaea villosa (Windham) Windham, (TEX 236024).

\section{SELAGINELLACEAE}

Selaginella lepidophylla (Hook. \& Grev.) Spring, (TEX 267413, 214647, 267428).

Selaginella peruviana (Milde) Hieron., (TEX 267520, 267522).

Selaginella pilifera A. Braun, (TEX 267539, 267542, 267543, 267545).

Selaginella rupincola Underw., (TEX 267601, 267608, 267614).

Selaginella wrightii Hieron., (TEX 214652).

\section{PINOPHYTA (GYMNOSPERMAE)}

\section{CUPRESSACEAE}

Cupressus arizonica Greene, 4294, 6725, 7993; 6995 BRIT; 8717 ANSM, BRIT.

Juniperus coahuilensis (Martínez) R.P. Adams, (TEX, 145156, 145157, 145168, 145185 , 145200).

Juniperus deppeana Steud., 2738, 2745, 5102, 5125; 6991 ANSM; 7168 ANSM; 7262 BRIT.

Juniperus monosperma (Engelm.) Sarg., 8215; 8345 ANSM, BRIT. 
Juniperus pinchotii Sudw., 8671 ANSM, BRIT.

Juniperus saltillensis M.T. Hall, (TEX 145803).

Juniperus scopulorum Sarg., 6621 ANSM.

EPHEDRACEAE

Ephedra aspera Engelm., 7049 TEX; (TEX 145955, 145969, 145990).

Ephedra pedunculata S. Watson, (TEX 142054, 142058).

Ephedra trifurca Torr., 5622 TEX; 7044 ANSM, BRIT, TEX; 7056 ANSM, TEX; (TEX 142088, 142104).

\section{PINACEAE}

Pinus engelmannii Carrière, 8586.

Pinus cembroides Zucc., 2746, 8427a .

Pinus leiophylla var. chihuahuana (Engelm.) Shaw, 2747; 3728 ANSM, BRIT; 8341; 8592

ANSM, BRIT, TEX; (TEX 144278).

\section{LILIOPSIDA (MONOCOTYLEDONEAE)}

\section{AGAVACEAE}

Agave lecheguilla Torr., 7980.

Agave parryi Engelm., 8292.

Agave potrerana Trel., (TEX 113757, 113760).

Agave scabra Salm-Dyck, 8698.

Yucca baccata Torr., 8409.

Yucca carnerosana (Trel.) McKelvey, 7202, 8696, 8697.

Yucca elata Engelm., 7239 ANSM, BRIT; (TEX 278589).

Yucca rigida (Engelm.) Trel., (TEX 154537).

Yucca rostrata Engelm. ex Trel., 6928 ANSM, BRIT, TEX; 8701 BRIT, TEX; 8976 BRIT.

\section{AMARYLLIDACEAE}

Hypoxis mexicana Schult., 7747 ANSM; 7793 ANSM, TEX; (TEX 113564).

Manfreda brunnea (S. Watson) Rose, 8369 ANSM, BRIT; 8401 TEX.

BROMELIACEAE

Hechtia glomerata Zucc., 7022.

Tillandsia recurvata (L.) L., 6996 BRIT. 


\section{COMMELINNACEAE}

Commelina coelestis Willd., 5802 ANSM, TEX.

Commelina dianthifolia Delile, 7696 ANSM, TEX; (TEX 110169).

Commelina erecta L. var. angustifolia (Michx.) Fernald, 5106 ANSM, BRIT, TEX; 5338 ANSM, TEX; 5812 ANSM, BRIT, TEX.

Commelina scabra Benth., (TEX 110491).

Gibasis linearis (Benth.) Rohweder, 5572, 7772 ANSM; 7384 ANSM, TEX; 7800 ANSM, TEX., (TEX 100628, 110632, 110634).

Gibasis pulchella (Kunth) Raf., 5204 ANSM, BRIT, TEX.

Tradescantia crassifolia Cav. var. glabrata C. B. Clarke, 7867 ANSM, BRIT, TEX.

Tradescantia pinetorum Greene, (TEX 113175).

Tradescantia wrightii Rose \& Bush var. glandulopubescens B.L. Turner, (TEX 113224).

Tradescantia sp., 4626 ANSM; 5540 TEX; 5605 TEX; 8025 TEX; 8223 BRIT; 8414 ANSM, TEX; 8431 ANSM, TEX; 8433 ANSM, BRIT, TEX.

\section{CYPERACEAE}

Bulbostylis juncoides (Vahl) Kük. ex Osten, 5552 CIIDIR; 8220 CIIDIR; 8295 CIIDIR; (NMC 44148, 57539; TEX 174092).

Cyperus aristatus L., (NMC 44140).

Cyperus esculentus L., 5507 BRIT, CIIDIR; 5857 CIIDIR; 8051 CIIDIR; 8074 CIIDIR; 8627 CIIDIR; 7944 BRIT, CIIDIR; (TEX 174330, 174344, 174345).

Cyperus fendlerianus Boeck., 7303 CIIDIR; 8278 CIIDIR, (NMC 48832).

Cyperus flavicomus Michx., (TEX 174400).

Cyperus manimae Kunth var. asperrimus (Liebm.) Kük., 7709 CIIDIR; 8783 CIIDIR.

Cyperus niger Ruiz \& Pav., 6447 CIIDIR; 7334 CIIDIR; 7338 CIIDIR; 8753 CIIDIR; (NMC 44147; TEX 174652, 174659).

Cyperus odoratus Kunth, (TEX 245892).

Cyperus cf. pallidicolor (Kük.) G.C. Tucker, 6165 CIIDIR; 7946 BRIT, CIIDIR; 7965 BRIT, CIIDIR; 8010 CIIDIR.

Cyperus rotundus Benth., (TEX 174771).

Cyperus seslerioides Kunth, 7791 BRIT, CIIDIR; 8532 CIIDIR.

Cyperus spectabilis Link, 7633 CIIDIR; 7659 CIIDIR; 7692 CIIDIR; 7697 CIIDIR; 8033 CIIDIR; 8225 BRIT; CIIDIR; 8256 CIIDIR; 8560 CIIDIR.

Cyperus sphaerolepis Boeck., 5260 CIIDIR; 5541 CIIDIR; 5575 CIIDIR; 6118 CIIDIR; 7304 CIIDIR; 7411a CIIDIR; 7692 CIIDIR; 7697 CIIDIR; 7931 CIIDIR; 8187 CIIDIR; 8210 CIIDIR; 8228 CIIDIR; 8253 CIIDIR; 8304 CIIDIR; 8542 CIIDIR. 
Cyperus squarrosus L., (TEX 174927).

Cyperus strigosus L., (NMC 44150).

Eleocharis arsenifera S. González, J. Tena \& T. Alarcón, (NMC 6560, 6888).

Eleocharis aff. macrostachya Britton, 8650 CIIDIR.

Eleocharis montevidensis Kunth, 7155 CIIDIR; 7166 CIIDIR; 7723 CIIDIR; (NMC 45860; TEX 245649).

Eleocharis parishii Britton, 5711; 5714 BRIT, CIIDIR; 7891 BRIT, CIIDIR; 8647 CIIDIR; (TEX 245651).

Fimbristylis cf. thermalis S. Watson, 5641 CIIDIR; 5647 CIIDIR, 5650 CIIDIR; 7914 BRIT, CIIDIR.

\section{IRIDACEAE}

Nemastylis tenuis var. pringlei (S. Watson) R.C. Foster, 7799 ANSM, BRIT, TEX.

Sisyrinchium scabrum Greene, (TEX 278307).

Sisyrinchium arizonicum Rothr *

Sisyrinchium tenuifolium Humb. \& Bonpl. ex Willd., 5644; 5646; (TEX 278769).

Tigridia pavonia (L.f.) DC., 9541.

\section{JUNCACEAE}

Juncus arcticus Willd. var. montanus (Engelm.) Balslev, (TEX 176894).

Juncus mexicanus Willd. ex Schult. \& Schult., 2601 ANSM.

Juncus nodosus L., 8641.

Juncus saximontanus A. Nelson, 7337 ANSM, TEX; 9055.

Juncus tenuis Willd., 7167 ANSM, BRIT, TEX; 7271 ANSM, TEX.

\section{LEMNACEAE}

Lemna gibba L. *

\section{LILIACEAE}

Allium glandulosum Link \& Otto, 5539 BRIT; 5568 ANSM; 5604 BRIT; 8362 BRIT; 8522 TEX; 8784 ANSM.

Allium kunthii G.Don, (TEX 112245).

Calochortus barbatus (Kunth) J. H. Painter, 8308 BRIT, TEX; 8510 ANSM; 8530a BRIT; 8539 BRIT; 9149.

Echeandia aff. chandleri (Greenm. \& C.H. Thomps.) Cruden, 7774 TEX.

Echeandia flavescens (Schult. \& Schult.) Cruden, 8040 BRIT, TEX; 8234; 8332 BRIT.

Milla biflora Cav., 5534, 5550 ANSM; 5560; 8038 TEX; (TEX 177678). 


\section{NOLINACEAE}

Dasylirion leiophyllum Engelm., 8499; 8661 ANSM; (TEX 111250).

Dasylirion texanum Scheele, 7205 ANSM, BRIT, TEX.

Nolina durangensis Trel., 4899 ANSM; 4942 ANSM, BRIT, TEX; 4971; 5095.

Nolina microcarpa S. Watson, 7731 ANSM; 8456; (TEX 278671).

Nolina texana S. Watson var. compacta (Trel.) I.M. Johnst., 5674; (TEX 278709).

\section{ORCHIDACEAE}

Dichromanthus cinnabarinus (La Llave \& Lex.) Garay, 9234; (TEX 111684; 111686).

Malaxis unifolia Michx., 8326 TEX.

\section{POACEAE}

Aegopogon cenchroides Humb. \& Bonpl. ex Willd., 8370 ANSM, BRIT, US; 8515 ANSM.

Aegopogon tenellus (DC.) Trin., 8735 US; 8768 BRIT; 8777 ANSM.

Agropyron arizonicum Scribn. \& J.G. Sm. *

Andropogon gerardi Vitman, (TEX 142619).

Aristida adscensionis L., 4708, 5216 NMC; 5437, 5692 NMC; 6076 US; 6360, 6720 BRIT,

US; 6809 NMC; 6832 NMC; 7609; 7645; 7784 ANSM, BRIT; 7840; 8057 BRIT; 8208;

8520 ANSM; 8523 NMC; 8609 NMC.

Aristida arizonica Vasey, 8424; 8453; 8458 NMC; 8462 BRIT; 8523 NMC; 8531 NMC; 8546

NMC; 8609 NMC; 8279 BRIT; 8792; 9029.

Aristida divaricata Willd., 7306 NMC; 8534 NMC; 8830 NMC; (NMC 48843; TEX 142891).

Aristida gypsophila Beetle f. gypsophilioides Allred \& Valdés-Reyna, 8577 BRIT, NMC.

Aristida havardii Vasey, 5369, 5393, 5420, 5831, 6825, 8200; 5431 BRIT, NMC; (TEX 142940).

Aristida pansa Wooton \& Standl. f. pansa, 5757NMC; 5764; 8070 NMC; 8227 NMC; 8298 NMC.

Aristida pansa var. dissita (I.M. Johnst.) Beetle, 7921 NMC; (TEX 142864).

Aristida purpurea Nutt. var. nealleyi (Vasey) Allred, 7494 NMC; 8480 NMC; 8473 BRIT, NMC; 8706 NMC; NMC 48830; (TEX 143034, 143043).

Aristida purpurea Nutt. var. perplexa Allred \& Valdés-Reyna, 6772 NMC; 8682 NMC.

Aristida purpurea Nutt. var. purpurea, 6830 BRIT, NMC; 6803 NMC; 6819 BRIT; (TEX 143147).

Aristida purpurea Nutt. var. wrightii (Nash) Allred, 8682 BRIT, NMC; 8685 BRIT, NMC. 
Aristida schiedeana Trin. \& Rupr. var. orcuttiana (Vasey) Allred \& Valdés-Reyna, 5777; 8394 BRIT; 8819 BRIT, NMC.

Aristida schiedeana Trin. \& Rupr. var. schiedeana, 7766; 8405 NMC; 8452 BRIT, NMC; 8531a NMC; 8565 NMC.

Aristida ternipes Cav. var. gentilis (Henrard) Allred, 8663 NMC; 5409 NMC; 5250 NMC; 5431 NMC; 8471 NMC; 8130 NMC; 8138 NMC; (NMC 48798).

Aristida ternipes Cav. var. ternipes, 5099 BRIT, NMC; 5257a BRIT, 7175 BRIT, NMC; 5839

NMC; 7630; 7783 NMC; 7841 NMC; NMC; 9075 NMC; 8833 NMC; 6186 NMC; 8201

NMC; (NMC 48851, 50810).

Arundo donax L. *

Bealia mexicana Scribn., (TEX 81706).

Blepharoneuron shepherdii (Vasey) P.M. Peterson \& Annable (TEX 82505).

Blepharoneuron tricholepis (Torr.) Nash, 8760 ANSM, BRIT, US; (TEX 143463).

Bothriochloa barbinodis (Lag.) Herter var. barbinodis, 4873 ANSM, US; 6155; 6163; 6476 ANSM; 6822 US; 7752 ANSM; 8202; 8448 ANSM.

Bothriochloa barbinodis (Lag.) Herter var. perforata (Trin. ex Fourn.) Gould, 8571 ANSM; 8684 BRIT.

Bothriochloa laguroides (DC.) Herter var. torreyana (Steud.) M. Marchi \& Longhi-Wagner, (TEX 143594).

Bothriochloa wrightii (Hack.) Henrard, 8437 ANSM, BRIT, US.

Bouteloua aristidioides (Kunth) Griseb., 5039 ANSM, BRIT; 5366; 5408 BRIT; 5486, 6068, 9066; 7834 ANSM; 8055 US.

Bouteloua barbata Lag., 5472 ANSM; 5656 BRIT; 7857 ANSM, BRIT.

Bouteloua breviseta Vasey, 8707 ANSM; (TEX 246359).

Bouteloua chondrosioides (Kunth) Benth. ex S. Watson, 5059, 5448 ANSM; 5565, 6102, 6488; 7780 ANSM; 9065 ANSM; (NMC 64239).

Bouteloua curtipendula (Michx.) Torr., 4860 BRIT, US; 4902; 4924, 6746; 6785 BRIT; 7649 ANSM, BRIT; 8203, 8296, 9071.

Bouteloua dactyloides (Nutt.) Columbus*** (reportado como Büchloe dactyloides (Nutt.) Engelm.

Bouteloua eriopoda (Torr.) Torr., 6745; 6786 US, 6821 ANSM, BRIT, NMC; 8097 BRIT, 8100 ANSM; 8193, 8930; 8949 ANSM, BRIT; 9031 NMC; 9056a US.

Bouteloua gracilis (Kunth) Lag., 2636, 5296, 5351, 5403; 5407 NMC; 5483; 5986 US; 6080 ANSM, BRIT; 6775 BRIT; 7641 ANSM; 8058; 8675 ANSM; 8945.

Bouteloua hirsuta Lag. ex Griffiths, 4943, 5082; 5261 BRIT, 5394, 5422; 5993 US; 6799, 6835, 7776, 8145, 8219, 8675.

Bouteloua parryi (E. Fourn.) Griffiths, 5669; 5672 ANSM, BRIT, US; (TEX 246568). 
Bouteloua simplex Lag., 7344 ANSM.

Bouteloua trifida Thurb., 5913a ANSM; (TEX 246550).

Bromus anomalus Rupr. ex E. Fourn., 8447 ANSM, US; 8718 ANSM; 8750a ANSM.

Bromus carinatus Hook. \& Arn., 8529 ANSM.

Bromus catharticus Vahl, 5842 US.

Bromus frondosus (Shear) Wooton \& Standl., (TEX 246939).

Bromus tectorum L., 5847 ANSM.

Cenchrus ciliaris L., 4830, 5398, 5426, 6703; 7117 ANSM; 7812 BRIT; 8997.

Cenchrus incertus M.A. Curtis, 4877; 7771 ANSM; (NMC 48826; TEX 245132).

Cenchrus myosuroides Kunth, 7065.

Chloris gayana Kunth, 7753 ANSM; 8275, 8318; 8371 ANSM, BRIT; 8611 ANSM; 8844 ANSM, BRIT.

Chloris submutica Kunth, 6069, 6077 US; 8043, 8083, 8123; 8918 US.

Chloris virgata Sw., 462, 5298, 5710; 5713 NMC; 5849 BRIT; 6349; 6728 NMC; 7307 ANSM, BRIT; 6831 BRIT, 8149, 8937 BRIT; (TEX 245408, 245376).

Cottea pappophoroides Kunth, 7859 BRIT, US; (TEX 245540).

Cynodon dactylon (L.) Pers., 4994, 5136, 6463; 6474 BRIT; 7119.

Dasyochloa pulchella (Kunth) Willd. ex Rydb., 5018, 5813, 6482, 7195, 7815, 8052, 8209, 9023.

Dichanthium annulatum (Forssk.) Stapf, 2771 ANSM; 5279.

Digitaria californica (Benth.) Henrard, 4812; 4874 BRIT; 5400, 5431a BRIT, TEX; 5549, 6795, 7064; 7883 ANSM, US; 8115a.

Digitaria ciliaris Retz., 7327 US; 7937 ANSM; 7987 US; (TEX 186322).

Digitaria pubiflora (Vasey) Wipff., 5255 ANSM, US; 5322; 5479 BRIT; 6787, 7496 US; 8197; 8644 BRIT; 8657 BRIT; (NMC 48833; TEX 186356, 186357).

Digitaria sanguinalis (L.) Scop., 5593.

Distichlis spicata (L.) Greene var. stricta (Torr.) Beetle, 5635; 5662 ANSM; 8638.

Echinochloa colona (L.) Link, 7336 ANSM, US; 8785 ANSM; (TEX 186499).

Echinochloa crusgalli (L.) P. Beauv. var. crusgallii, 5704 US; 5709 ANSM; 5712 BRIT; 8530 ANSM, BRIT, US; 8778 ANSM; (TEX 186576).

Echinochloa crusgalli (L.) P. Beauv. var. zelayensis (Kunth) Hitchc., (TEX 186592).

Echinochloa crus-pavonis (Kunth) Schult., 5800 ANSM, BRIT, TEX.

Echinochloa oplismenoides (E. Fourn.) Hitchc., (TEX 186613).

Echinochloa polystachya (Kunth) Hitchc., 8216 ANSM, US.

Elyonurus barbiculmis Hack., 8226, 8293; 8536 ANSM; 8574 ANSM, BRIT, NMC, US; 8678 ANSM; 8801 US.

Enneapogon desvauxii P. Beauv., 5219 NMC; 6169; 6721 ANSM; 7921 NMC; 8103a US; 8955; 9064 US. 
Eragrostis cilianensis (All.) Vignolo ex Janch. 5494; 5522 US; 6082 ANSM; 6784 US; 6838; 6852 BRIT; 9041; (TEX 186821, 186822).

Eragrostis curvula (Schrad.) Nees, 7779 ANSM, US.

Eragrostis erosa Scribn. ex Beal, (TEX 186900).

Eragrostis intermedia Hitchc., 4918 ANSM, US; 5266 ANSM; 5564 ANSM, US; 6084 ANSM, US; 6851 ANSM, US; 8230 ANSM, US; 8250 ANSM; 8689 BRIT; (NMC 48828, 50826; TEX 186998, 187002).

Eragrostis lehmanniana Nees, 9000 US; 9057 US; (TEX 188011).

Eragrostis lugens Nees, 4958 US.

Eragrostis mexicana (Hornem.) ssp. virescens (J. Presl) S. D. Koch \& Sánchez Vega, 4876 US; 5140 ANSM, US; 5151; 5307; 5313 BRIT, US; 5341 ANSM, BRIT, US; 5357; 5615 BRIT; 5828 ANSM, BRIT, US; 5875 BRIT; 6702 ANSM, US; 7189 US; 7292 US; 7325 ANSM, US; 7601 US; 7666 ANSM; 7782 ANSM; 8085 ANSM; 8103 BRIT, US; 8110 US; 8147 ANSM, BRIT; 8752 ANSM; 8920; 8927 ANSM.

Eragrostis obtusiflora (E. Fourn.) Scribn., 7097 ANSM, BRIT, US.

Eragrostis palmeri S. Watson, 4878 ANSM, US; 7962 ANSM, US; 8023 ANSM, US; 8621 ANSM, US; 8826 BRIT, US; 8834 ANSM, US; 8947 ANSM, US.

Eragrostis pectinacea (Michx.) Nees var. miserrima (E. Fourn.) Reeder, 5515 US; 5523; 5607 US; 6473 US; 6862 BRIT, US; 7610 US; 8101 ANSM; 8129 US; 8176 US; 8179 US; (NMC 48825; TEX 188168, 188169, 188170, 188171, 188175, 188180).

Eragrostis pilosa (L.) P. Beauv., 8124 US; 9027 ANSM.

Eragrostis superba Peyr., 4149; 5844 ANSM, BRIT.

Eriochloa acuminata (J. Presl) Kunth var. acuminata, 5841 ANSM, US; 6101; 7943 ANSM, US; 8066; (TEX 188322, 188323).

Eriochloa acuminata (Presl) Kunth var. minor (Vasey) R.B. Shaw, 8122 ANSM.

Eriochloa aristata Vasey var. aristata, 6151 US; 6162, 6168 US; 6174 ANSM; 7634 ANSM; 8171.

Eriochloa lemmonii Vasey \& Scribn. var. lemmonii, 8570 US.

Eriochloa polystachya Kunth, 5843 ANSM, BRIT, US; (TEX 188347).

Eriochloa punctata (L.) Desv. ex Ham., 5591 US; 5612.

Erioneuron nealleyi (Vasey) Tateoka, 7620a.

Hackelochloa granularis (L.) Kuntze, 6135 ANSM; 6172.

Heteropogon contortus P. Beauv. ex Roem. \& Schult., 4914; 4956 BRIT, US; 4995 NMC; 5096; 5421 BRIT; 6810 BRIT, NMC; 7781; 8281 ANSM, BRIT; 8676 BRIT; 8968; (NMC 8905).

Hilaria belangeri (Steud.) Nash, 8290; 8479.

Hilaria swalleni Cory, 8198 ANSM. 
Hordeum pusillum Nutt. *

Leptochloa dubia (Kunth) Nees, 5531; 5781; 5788 US; 5846 US; 6834; 7636 ANSM, BRIT, 8196; 8967.

Leptochloa fusca (L.) Kunth ssp. fascicularis (Lam.) N. Snow, 5705 BRIT, US; 7335 US.

Lycurus phleoides Kunth, 4966 BRIT; 5470 US; 5484 ANSM; 5766; 5778 BRIT; 6345; 6464; 7295 US; 7778.

Melinis repens (Willd.) Zizka, 5283; 5315; 6836; 7053; 7775; 8857 ANSM.

Microchloa kunthii Desv., 9273; (NMC 48829).

Muhlenbergia aff. xerophila C. O. Goodding, 8674 US.

Muhlenbergia arenacea (Buckley) Hitchc., 8568 ANSM, BRIT, US.

Muhlenbergia arenicola Buckley, (TEX 81680).

Muhlenbergia asperifolia (Nees \& Meyen ex Trin.) Parodi, (TEX 81697, 81700, 81703).

Muhlenbergia brevis C.O. Goodding, (TEX 82659).

Muhlenbergia crispiseta Hitchc., 7662b.

Muhlenbergia cuspidata (Torr.) Rydb., 8334 ANSM, US.

Muhlenbergia depauperata Scribn., (TEX 81744).

Muhlenbergia divaricata Kunth, 7306 NMC.

Muhlenbergia dubia E. Fourn., 8317 US; 8526 US; 8541 US; 8545 ANSM.

Muhlenbergia eludens C. Reeder, 7605a; (TEX 81809).

Muhlenbergia emersleyi Vasey, 6837 ANSM, 6864; 7202 BRIT; 8284 ANSM; 8576, 8659 ANSM, NMC; 8814 ANSM, NMC; 8898a; 8975 BRIT, US; (TEX 81821, 81825, 81829). Muhlenbergia flavida Vasey, 7595 BRIT, US.

Muhlenbergia fragilis Swallen, 8732 ANSM, BRIT, US; 8899 BRIT, US, 8929 US; (TEX 81873, 81877).

Muhlenbergia glauca (Nees) B. D. Jacks. 8022 ANSM, US; 8672 ANSM, US.

Muhlenbergia implicata (Kunth) Trin.**

Muhlenbergia lucida Swallen, 8303 ANSM, US; 8597 ANSM.

Muhlenbergia macroura (Kunth) Hitchc., 8351 ANSM, BRIT, US; 8543 US.

Muhlenbergia majalcensis P.M. Peterson, 7333 ANSM, US; 8507 ANSM, BRIT, US.

Muhlenbergia minutissima (Steud.) Swallen, 8410b; 8750 ANSM, BRIT, US.

Muhlenbergia montana (Nutt.) Hitchc., 7274 BRIT; 8307 BRIT, TEX; 8346; 8393 BRIT; 8455 BRIT; 8525 ANSM; 8537 US; 8544 BRIT; 8593 ANSM, BRIT, US; 8607 BRIT.

Muhlenbergia monticola Buckley ***

Muhlenbergia palmeri Vasey ex S. Watson, 8604 ANSM, BRIT, US.

Muhlenbergia pauciflora Buckley, 5786 US; 7655 ANSM, US; 8340 US; 8559 ANSM, US; 8572 ANSM; 8578 ANSM, US; (TEX 82199).

Muhlenbergia peruviana (P. Beauv.) Steud., 8412a; (TEX 82697). 
Muhlenbergia polycaulis Scribn., (TEX 82214).

Muhlenbergia porteri Scribn. ex Beal, 5736 US; 5758 US; 5808 US; 8485 ANSM; 8550 ANSM, BRIT, US; 8811, 8993 ANSM, BRIT, US.

Muhlenbergia pringlei (Scribn.) Scribn., (TEX 82693).

Muhlenbergia ramulosa (Kunth) Swallen, (TEX 82651).

Muhlenbergia repens (J. Presl) Hitchc., 9035 BRIT, US.

Muhlenbergia rigens (Benth.) Hitchc., 6425 ANSM, BRIT, US; 8262 NMC, US; 8886a US.

Muhlenbergia rigida (Kunth) Kunth, 8572 BRIT, US; 8612 ANSM, BRIT, US; 8822 ANSM,

NMC, US; 8871 ANSM; (TEX 82370).

Muhlenbergia robusta (E. Fourn.) Hitchc., 8454 ANSM, US; 8691 US; 8748 ANSM, US.

Muhlenbergia spiciformis Trin., 8513a US; 8535 BRIT; 8540 ANSM, US.

Muhlenbergia strictior Scribn. ex Beal **

Muhlenbergia tenuifolia (Kunth) Kunth, 8519c; 8348 ANSM, BRIT, US; (NMC 52570; TEX 82086, 82090; 82576).

Muhlenbergia utilis (Torr.) Hitchc., 8645 ANSM, BRIT, US.

Panicum antidotale Retz., 8563 US; (TEX 82596).

Panicum bulbosum Kunth, 7270 US; 7367 BRIT; 7615; 7660 ANSM; 7950 ANSM, BRIT; 8263 BRIT; 8294 US; 8467 ANSM; (TEX 82970).

Panicum hallii Vasey, 5845 ANSM, BRIT, US; 5850a, 6948; 7092; (NMC 50825; TEX 83149).

Panicum hirticaule J. Presl, 5124, 5471 BRIT; 5806 ANSM, BRIT, TEX; 5874 ANSM; 7803

ANSM; 7807 US; 7929 US; 8048, 8113; 8565a BRIT; 8846, 8922 ANSM, US.

Panicum obtusum Kunth, 5199, 5240 BRIT, 5482 US; 5532 BRIT; 5723; 7890 BRIT, 8050;

8146 ANSM; 8847; 8916 BRIT.

Pappophorum vaginatum Buckley, (TEX 83558).

Paspalum pubiflorum Rupr. ex E. Fourn., (TEX 83915, 83916).

Paspalum vaginatum L., 5582 ANSM, US; 7875 US; 8624 US; (TEX 83687); 8265 US.

Peyritschia deyeuxioides (Kunth) Finot, 8538 ANSM.

Phalaris caroliniana Walter*

Piptochaetium fimbriatum (Kunth) Hitchc., 7629, 7708; 7797 ANSM, BRIT.

Pleuraphis mutica Buckley, 5614 BRIT; 5701 ANSM; 6336, 8928; (TEX 80803, 80810).

Polypogon elongatus Kunth (TEX 75379).

Polypogon monspeliensis (L.) Desf., 7120 ANSM, BRIT, US; 8643; (TEX 75386).

Polypogon viridis (Gouan) Brelstr., 7330 ANSM, BRIT, US; (TEX 142463).

Schizachyrium cirratum Wooton \& Standl. var. cirratum, (TEX 75515, 75519).

Schizachyrium sanguineum var. brevipedicellatum (Beal) S.L. Hatch, (ARIZ, MEXU, NMC, TEX 75538). 
Schizachyrium sangineum (Retz.) Alston var. sanguineum, 8229 ANSM, BRIT, US; 8315; 8449 US; 8679 ANSM, US.

Schizachyrium scoparium (Michx.) Nash, 7256 ANSM, US; 7665 US; 7667 ANSM, BRIT; 7688; 8313 ANSM, BRIT; 8392 ANSM; 8606 ANSM.

Scleropogon brevifolius Phil., 8054 ANSM, BRIT, US; 8576a; 8664 ANSM, BRIT; 9003 NMC.

Setaria adhaerens (Forssk.) Chiov. (TEX 235743).

Setaria grisebachii E. Fourn., 5750 US; 5769 US; 6429 US; 6432 US; 7208 ANSM, BRIT, US; 7602 ANSM, BRIT, US; 7825 ANSM, BRIT, US; 8956 ANSM, US; (TEX 75870).

Setaria leucophylla (Scribn. \& Merr.) K. Schum., 4799; 4875 ANSM, BRIT, NMC, US; 5171

BRIT, US; 5349 ANSM; 5581 ANSM, BRIT; US; 5829 ANSM; 7851 US; 8804 ANSM, US; 8933 ANSM, US.

Setaria macrostachya Kunth, 5861 ANSM, BRIT, US; 8951 BRIT, US.

Setaria parviflora (Poir.) Kerguélen, 6704 ANSM, BRIT, US; 7656 ANSM; (TEX 75787).

Setaria scheelei (Steud.) Hitchc., 8255 ANSM, US.

Setaria viridis (L.) P. Beauv., 4875; 5160 ANSM, US; 5368 US; 5370 ANSM; 5670 US; 7125

ANSM, US; 7668 ANSM; 7809a US; 8092 ANSM, US; 8575 ANSM, US; 8853 US; 8854

ANSM, BRIT.

Sorghum halepense (L.) Pers., 5100, 5110, 8172.

Sporobolus airoides (Torr.) Torr., 5702 ANSM; 8652.

Sporobolus contractus Hitchc., 5353 ANSM, US.

Sporobolus cryptandrus (Torr.) A. Gray, 5488, 5642; 8099 ANSM, BRIT, US.

Sporobolus pulvinatus Swallen ***

Sporobolus pyramidatus (Lam.) Hitchc., 5696 US, 6396 ANSM.

Trachypogon spicatus (L.f.) Kuntze, 8285 ANSM, US; 8558; 8690 ANSM, BRIT, US; (NMC 64240).

Tragus berteronianus Schult., 5281; 5306 BRIT; 5352; 5535 BRIT; 6384; 6833; 7811 BRIT; 8044 ANSM; (NMC 48824).

Tridens albescens (Vasey) Wooton \& Standl., 5754, 5765; 5783 ANSM; 5910 US; 6744 US; 6783 ANSM; 6801; 8852 US.

Urochloa arizonica (Scribn. \& Merr.) Morrone \& Zuloaga, 4870 BRIT; 4913; 5188; 5277 ANSM, US; 5285; 5295.

Urochloa fusca (Sw.) B. F. Hansen \& Wunderlin, 4868 ANSM, BRIT, US; 5866 ANSM, BRIT; 5882 US; 8562.

Urochloa meziana (Hitchc.) Morrone \& Zuloaga, 6138 US; 7764 ANSM, BRIT; 8192 ANSM; 8251 BRIT.

Urochloa plantaginea (Link) R.D. Webster, 7924 US. 


\section{PONTEDERIACEAE}

Heteranthera limosa (Sw.) Willd., 5594 ANSM.

\section{POTAMOGETONACEAE}

Potamogeton foliosus Raf. *

\section{TYPHACEAE}

Typha latifolia L., 7894 BRIT.

\section{MAGNOLIOPSIDA (DICOTYLEDONEAE)}

\section{ACANTHACEAE}

Anisacanthus puberulus (Torr.) Henr. \& E.J. Lott, 7048 ANSM, BRIT, TEX; (TEX 105798, 105808).

Carlowrightia arizonica A. Gray, (TEX 87685).

Carlowrightia lesueuri Henr. \& T. F. Daniel, (TEX 87722).

Carlowrightia mexicana Henr. \& T. F. Daniel, (TEX 87731).

Carlowrightia ovata A. Gray *

Carlowrightia texana Henr. \& T. F. Daniel *

Dyschoriste linearis (Torr. \& A. Gray) Kuntze, 4663; 7686 ANSM.

Dyschoriste schiedeana (Nees) Kuntze var. decumbens (A. Gray) Henr., 4296; 5025 TEX; 5259, 264, 6145; 6616 BRIT; 7075 BRIT; 7204; 8502 ANSM; 8504; 8964 BRIT (NMC 48788, 48876).

Elytraria imbricata (Vahl) Pers., 5010 ANSM, NMC; 5016, 5195; 5859 NMC; 7029, 7045;

7871 BRIT; 8549, 8655, 9446.

Tetramerium nervosum Nees, 7846 ANSM, BRIT, TEX; (TEX 89392).

\section{AMARANTHACEAE}

Alternanthera caracasana Kunth, 7942; 9126.

Alternanthera polygonoides (L.) R. Br., 2478 ANSM.

Amaranthus palmeri S. Watson, 4807 ANSM, BRIT, TEX; 5046, 5049, 5251; 5504 ANSM;

6793 ANSM; 7406; 7646 ANSM; 7801 ANSM, BRIT, TEX; (TEX 173541).

Amaranthus powellii S. Watson, 7247 *

Amaranthus torreyi (A. Gray) Benth. ex S. Watson, (TEX 191533).

Froelichia arizonica Thornber ex Standl., 4609; 4822 ANSM; 4865; 5197 TEX; 5373; 6484, 6870 ANSM; 8108 BRIT; 8280; 8972 ANSM. 
Froelichia gracilis (Hook.) Moq., 7301 ANSM, BRIT.

Froelichia interrupta (L.) Moq., 5543; 5553 TEX.

Gomphrena nitida Rothr., 4825 TEX; 5323 TEX; 5462; 6752 ANSM; (NMC 48789).

Gomphrena serrata L., 5462; (TEX 173773).

Gomphrena sonorae Torr., 6452 TEX; 6453 ANSM; 8667; (TEX 173856).

Guilleminea densa (Willd.) Moq. var. agreggata Uline \& Bray, 4982 ANSM; 7289 BRIT; 7592 BRIT; 7936; 8072 NMC.

Guilleminea lanuginosa (Poir.) Hook. var. lanuginosa, 7978 BRIT; 8803, 6467.

Iresine diffusa Humb. \& Bonpl. ex Willd., 5456; 6137a ANSM; 6854 ANSM, TEX; 7653 TEX; 7661a.

Iresine heterophylla Standl., 4643; (NMC 63089).

Iresine leptoclada (Hook.) Henr. \& S. D. Sundb., (TEX 49685).

Tidestroemia lanuginosa (Nutt.) Standl. var. lanuginosa, 6737 ANSM, BRIT, TEX.

Tidestroemia suffruticosa (Torr.) Standl., 5145 TEX; 5194; 7821 ANSM.

\section{ANACARDIACEAE}

Rhus aromatica Aiton, (TEX 103308, 103335).

Rhus microphylla Aiton, 2475, 5213, 5768, 6797; 6931 ANSM; 7031 BRIT.

Rhus trilobata Nutt. ex Torr. \& A. Gray, 6099; 7356 ANSM, BRIT; 7598.

Rhus virens A. Gray var. choriophylla L.D. Benson, 4937 ANSM; 7000; 7969 NMC; 8863; (TEX 103742, 103743).

Rhus virens A. Gray var. virens, (TEX 103783).

\section{APIACEAE}

Aletes acaulis (Torr.) J. M. Coult. \& Rose *

Cyclospermum leptophyllum (Pers.) Sprague ex Britton \& P. Wilson,7173 ANSM, TEX; 9139. Eryngium heterophyllum Engelm., 6115 NMC; 6119 BRIT; 8140 ANSM; 8382 BRIT; 8632; 8715 ANSM; 8759 ANSM; 8827; (TEX 162788, 162802).

\section{APOCYNACEAE}

Apocynum androsaenifolium L. *

Haplophyton crooksii (L. Benson) L. Benson, 7855 ANSM, BRIT, TEX; (TEX 251847, 251859).

Mandevilla foliosa (Müll. Arg.) Hemsl., 6999 ANSM, BRIT, TEX; 7296 BRIT, TEX; 7614 TEX; 7786 TEX; 7968 TEX; 8246 TEX; (NMC 59611).

Telosiphonia hypoleuca (Benth.) Henr., 4944 ANSM, TEX; 5031; 5536 BRIT; 5567 BRIT; 8218, 8688; 8832 BRIT; (NMC 52816; TEX 250283). 
Telosiphonia macrosiphon (Torr.) Henr., 4615, 5120; 5158 TEX; 5280 ANSM; 5912 ANSM; 6749; 7137 ANSM; 7870 TEX; (TEX 250311, 250312).

ARALIACEAE

Aralia humilis Cav., 9443.

ARISTOLOCHIACEAE

Aristolochia wrightii Seem. var. texana I.M. Johnst., 5196 BRIT; 7047 ANSM, BRIT.

\section{ASCLEPIADACEAE}

Asclepias asperula (Decne.) Woodson, 2464, 2465, 4687; (TEX 192109).

Asclepias brachystephana Torr., 2634; 5275 ANSM, BRIT; 5287, 5390; 7077 ANSM, BRIT;

(TEX 184381, 192141).

Asclepias elata Benth., 7354 BRIT; 7677 BRIT; 8423; 8528 BRIT 8818.

Asclepias linaria Cav., 5080 BRIT; 7973; 8032 ANSM.

Asclepias macrotis Torr.,(TEX 192153, 192158, 192161).

Asclepias nummularia Torr., 7446; 7673 ANSM; 9150.

Asclepias oenotheroides Schltdl. \& Cham., 5696 ANSM; 7903 a.

Asclepias subverticillata (A. Gray) Vail, 5699 ANSM, BRIT; 7884 BRIT.

Asclepias texana A. Heller *

Funastrum crispum (Benth.) Schltr., 5436 ANSM.

Funastrum cynanchoides (Decne.) Schltr. var. hartwegii (Vail) Krings, 2765; 7105 ANSM, BRIT; 7934 BRIT; 8180 TEX; 8799; 8988 ANSM.

Matelea chihuahuensis (A. Gray) Woodson, 8429 TEX.

Matelea parvifolia (Torr.) Woodson, 7224 ANSM, BRIT, TEX.; 9152.

Metastelma barbigerum Scheele var. breviflorum Shinners, 7964 BRIT.

\section{ASTERACEAE}

Achillea millefolium L., 7372 BRIT; 7724 ANSM.

Acourtia nana (A. Gray) Reveal \& R.M. King, 5751 ANSM, BRIT; 8045 TEX.

Acourtia parryi (A. Gray) Reveal \& R.M. King *

Acourtia wrightii (A. Gray) Reveal \& R.M. King, 4659 ANSM; 4806; 4859 ANSM; TEX;

5392 US; 5416 ANSM; 6639 BRIT; 7096 BRIT; 7227 ANSM, BRIT, NMC, US; 8493

BRIT; 8580 BRIT; 5416.

Adenophyllum cancellatum (Cass.) Villarreal, 4641; 5465 ANSM; 5526; (NMC 56386) 6750

ANSM; 6788 TEX; 7768 ANSM, BRIT; (TEX 58086, 58092, 58094, 58095).

Ageratina choricephala (B.L. Rob.) R.M. King \& H. Rob. * 
Ageratina havanensis (Kunth) R.M. King \& H. Rob. *

Ageratina hyssopina (A. Gray) R.M. King \& H. Rob., (TEX 51870).

Ageratina lemmonii (B.L. Rob.) R.M. King \& H. Rob., 8907 TEX.

Ageratina rothrockii (A. Gray) R.M. King \& H. Rob., 4668, 4900, 5014; 6824 US; 8086 ANSM, NMC.

Ageratina wrightii (A. Gray) R.M. King \& H. Rob., 6194 TEX; 6518 TEX; 6566 TEX; 6805

ANSM, BRIT; 8669 ANSM, BRIT, NMC; (TEX 53085, 53090, 53094).

Ageratum corymbosum Zuccagni, 4651; 5460 BRIT; 5853 BRIT; 5856 BRIT; 8231 ANSM; 8816; 9052 NMC, US; (TEX 53194, 53197, 53203).

Almutaster pauciflorus (Nutt.) Á. Löve \& D. Löve, 5640 ANSM, TEX; 5658 TEX.

Ambrosia acanthicarpa Hook., (TEX 120803, 120807).

Ambrosia confertiflora DC., 5797 ANSM, TEX, US; 6711 BRIT, TEX; 6759 ANSM, TEX; 8168 BRIT, TEX; 8268, 8808 US; 9021; (TEX 120999).

Ambrosia psilostachya DC., 7719 TEX; 8633 TEX; (TEX 141754).

Aphanostephus ramosissimus (L.) DC. var. humilis (Benth.) Turner \& Birdsong, 4886; 5043

TEX; 5272 BRIT; 5278, 5364, 6433, 6442, 6485; 6736 US; 6895 TEX; 7041, 7093, 7094, 7109; 7115 BRIT, TEX; 7203 TEX; 7594 TEX; 7903 BRIT, NMC, TEX; 7977 TEX; (TEX 67050, 67052).

Artemisia campestris L. (TEX 62273).

Artemisia carruthii Alph. Wood ex Carruth, 8269; 8469 ANSM, BRIT; 8969 ANSM, TEX; 9046 US.

Artemisia caudata Michx., 6449 ANSM, TEX, US.

Artemisia dracunculus L., (TEX 62284).

Artemisia filifolia Torr., (TEX 62292, 62298).

Artemisia ludoviciana Nutt., 6097, 6178; 6510 TEX; 6639 TEX; 6663 TEX; 6853 ANSM;

7679 ANSM; 8011 ANSM, TEX; 8254; 8465 US; 8821; 8872 ANSM; 8908 BRIT; (TEX 62345, 62346).

Artemisia pringlei Greenm. (TEX 62435, 62438, 62441).

Aster pauciflorus Nutt., 5434 ANSM.

Aster praealtus Poir., 5643, 5707.

Aster subulatus Michx., 5805 ANSM, US.

Baccharis bigelovii A. Gray, 8781a; (TEX 68216, 68218).

Baccharis neglecta Britton, 5048 BRIT, TEX; 5694 ANSM, TEX.

Baccharis pteronioides DC., 6945 TEX; 7634 ANSM, TEX; 7680 TEX ; (TEX 68595, 68603).

Baccharis salicifolia (Ruiz \& Pav.) Pers., 5198 ANSM, TEX; 6562 TEX; 7506 TEX; 7299

ANSM, TEX; 7941 ANSM. 
Baccharis salicina Torr. \& A. Gray (TEX 68851).

Baccharis sulcata DC., 5886 TEX; 5906 TEX.

Baccharis wrightii A. Gray, (TEX 69178, 69180).

Bahia absinthifolia Benth. var. absinthifolia, 2627; 5818 ANSM; 6283 TEX; 6381 ANSM; 6943 TEX; 7839; 8702 US.

Bahia absinthifolia Benth. var. dealbata A. Gray, (TEX 55615, 55618).

Bahia dissecta (A. Gray) Britton, 6060 TEX; 7836 TEX; 8900 ANSM, TEX.

Bahia pedata A. Gray, (TEX 55722).

Baileya multiradiata Harv. \& A. Gray, 2466, 2467, 5130, 5193; 5538; 6735 ANSM; 7214; 4191 ANSM.

Bartlettia scaposa A. Gray, (TEX 55909, 55911, 55913) *

Berlandiera lyrata Benth., 4157 ANSM; 6859 TEX; 7076 ANSM, BRIT; 8080.

Bidens aurea (Aiton) Sherff (NMC 52629; TEX 122065).

Bidens ferulifolia (Jacq.) DC., 8630 ANSM, BRIT, TEX; 8828 TEX.

Bidens leptocephala Sherff (TEX 121893, 121894).

Bidens odorata Cav. var. odorata, 5840 ANSM; 6074 ANSM; 6093 NMC; 7313 US; 7322

TEX; 7374 ANSM; 7616; 7628 US; 7763 ANSM; (TEX 122660).

Bidens schaffneri (A. Gray) Sherff var. wrightii (A. Gray) Melchert, (TEX 121978).

Brickellia amplexicaulis B.L. Rob., 6713 ANSM, BRIT, TEX.; 7599 ANSM; (TEX 53766).

Brickellia betonicifolia (Torr. \& A. Gray) A. Gray, (TEX 53795).

Brickellia californica A. Gray, 7864 TEX, US.

Brickellia coulteri A. Gray var. brachiata (A. Gray) B.L. Turner, (TEX 53883).

Brickellia eupatorioides (L.) Shinners var. chlorolepis (Wooton \& Standl.) B.L. Turner, 2762, 7005, 7061; 7113 TEX; 7228 ANSM, BRIT; 8974 TEX; 9038 US.

Brickellia floribunda A. Gray, 8862; 8872a ANSM, TEX, US; TEX 114104, 114105).

Brickellia laciniata A. Gray, 2468; 8962 ANSM, BRIT, NMC, TEX; 9079 ANSM, BRIT; (TEX 114230, 114295).

Brickellia lemmonii A. Gray var. conduplicata (B.L. Rob.) B.L. Turner, 8355 US; 8594 TEX; 8894 ANSM, BRIT, TEX, US.

Brickellia lemmonii A. Gray var. lemmonii, (TEX 114366, 114367, 114368).

Brickellia odontophylla A. Gray *

Brickellia rusbyi A. Gray, 6124; 6278a.

Brickellia simplex A. Gray, (TEX 114753, 114759).

Brickellia spinulosa (A. Gray) A. Gray, (NMC 48871; TEX 114771, 114774).

Brickellia subuligera (Schauer) B.L. Turner, 8850 ANSM, BRIT, TEX.

Brickellia tomentella A. Gray, 6481 ANSM, US. 
Brickellia venosa (Wooton \& Standl.) B.L. Rob., 4695; 6811 TEX; 6812 ANSM, BRIT, NMC; 8817 BRIT, NMC; 8960 ANSM, BRIT, US; (TEX 114881).

Brickellia veronicaefolia (Kunth) A. Gray var. petrophila (B.L. Rob.) B.L. Rob., 9050 ANSM, BRIT, TEX.

Brickellia wislizeni A. Gray, (NMC 56550; TEX 115027, 115031, 115034).

Carminatia tenuiflora DC., 6175 ANSM, BRIT, TEX, US.

Carphochaete wislizeni A. Gray, 7694 ANSM, TEX, US; 8300; 8400 US

Chaetopappa bellioides (A. Gray) Shinners, (TEX 69186, 69187, 69189).

Chaetopappa ericoides (Torr.) G.L. Nesom, 7013 TEX; 7141 ANSM, BRIT, TEX; 7758 TEX; 7813 TEX; (TEX, 69293, 69297).

Cirsium ochrocentrum (A. Gray) A. Gray, 4165 ANSM; 7196 ANSM, BRIT, TEX; (TEX 64545).

Cirsium texanum Buckley *

Cirsium undulatum (Nutt.) Petr., (TEX 64688, 64691).

Conoclinium betonicifolium (Mill.) R.M. King \& H. Rob. var. integrifolium (A. Gray) Patt., (TEX 115842, 115843, 115844).

Conoclinium dissectum A. Gray, 6824 TEX, BRIT; 8086; (TEX 115889, 115890).

Conyza canadensis (L.) Cronquist, 2661 ANSM; 7979 TEX; 8618 US; 8837 US.

Conyza coulteri A. Gray, 4819, 4884; 5464 ANSM; 7895 ANSM, TEX, US; 8166; 8721 US; 8738 TEX.

Conyza sophiifolia Kunth, 4879 US; 7773a; 8249, 8805; 8934 TEX; 9039 ANSM.

Coreopsis tinctoria Nutt., 8635 ANSM, BRIT, TEX; (TEX 123872, 123873, 123876, 123879).

Cosmos palmeri B.L. Rob., 8331 TEX; (TEX 124121).

Cosmos parviflorus (Jacq.) Pers., 8330 TEX; 8779 ANSM, TEX.

Dahlia coccinea Cav., 8327 ANSM, BRIT, TEX; (TEX 124142, 124145, 124149).

Dahlia sherffii P.D. Sorensen, 7735 ANSM, US.

Diaperia verna (Raf.) Morefield *

Dyssodia papposa (Vent.) Hitchc., 6204 TEX; 6600 BRIT; 6817a; 8839 TEX; 8917; 9040 BRIT.

Encelia scaposa (A. Gray) A. Gray, 6965 TEX.

Erigeron coronarius Greene, 7200 TEX; 7727 TEX; 7745 TEX; 7755 TEX; 8239 TEX; 8261 TEX; 8887 TEX; (NMC 48775).

Erigeron delphinifolius Willd., 7617 ANSM, BRIT; 7695 ANSM, BRIT; 7617.

Erigeron divergens Torr. \& A. Gray, 5609 TEX; 5884 TEX; (NMC 52430; TEX 70485, 70492).

Erigeron flagellaris A. Gray, 2471 ANSM; 7149 TEX; 7162 ANSM, TEX.

Erigeron inoptatus A. Gray, (TEX 70810). 
Erigeron metrius S.F. Blake, (TEX 137128).

Erigeron modestus A. Gray, 2763; (TEX 137208).

Erigeron oreophilus Greenm., 7268 TEX, 7357 TEX; 7703 TEX; 8239 TEX; 8261 TEX; 8887 TEX; 8913 TEX.

Erigeron pubescens Kunth *

Erigeron strigulosus Greene, (TEX 137600).

Erigeron versicolor (Greenm.) G.L. Nesom, 7291 TEX.

Erigeron wislizeni (A. Gray) Greene var. wislizeni, (TEX 71141, 71144, 71147).

Flaveria chloraefolia A. Gray, 4794 ANSM; (TEX 56695, 141982).

Flaveria trinervia (Spreng.) C. Mohr, 6405 ANSM, TEX; (TEX 56826, 56827, 56828, 56830).

Florestina pedata (Cav.) Cass., 6731; 6761 ANSM, BRIT; 6776 US.

Florestina tripteris DC., 4670; 5740 ANSM, BRIT; 7098; 7819 ANSM, BRIT, TEX; 8986 US.

Flourensia cernua DC., 5698, 6726; 7019 BRIT.

Gaillardia pinnatifida Torr., 5384; 5412 US; 5413; 6733 ANSM, BRIT, 7087 TEX; 8063; 8151 ANSM, TEX, US.

Gaillardia pulchella Foug. var. pulchella, (NMC 52839; TEX 57343, 57344).

Gaillardia turneri Averett \& A.M. Powell, (TEX 57375, 57376, 57379, 57381).

Galinsoga parviflora Cav., 6098 TEX; 6709 TEX; 7284 TEX; 7332 TEX; 7600; 7635 TEX; 8311 TEX; 8601 BRIT, TEX; (TEX 125347).

Gnaphalium arizonicum A. Gray, 8884 TEX.

Gnaphalium canescens DC., 8824 ANSM, BRIT, TEX, US.

Gnaphalium pringlei A. Gray, 8724 TEX; 8877 ANSM, TEX.

Gnaphalium stramineum Kunth, 7288 TEX; 7321 BRIT, TEX, US; 7717 ANSM, BRIT, TEX; 7743 TEX; 8626 ANSM, BRIT, TEX.

Gnaphalium viscosum Kunth, 8260 TEX.

Grindelia arizonica A. Gray var. arizonica, 8648 ANSM, BRIT, TEX; 7885 BRIT, TEX; (TEX 71610).

Grindelia eligulata (Steyerm.) G.L. Nesom *

Grindelia oxylepis Greene, (TEX 136652, 136655).

Gutierrezia conoidea (Hemsl.) M.A. Lane, 8376 BRIT, TEX, US; 8782 BRIT, TEX; (TEX 71230).

Gutierrezia microcephala (DC.) A. Gray, 8161 TEX; 6397 TEX; 9004 BRIT, TEX; (TEX 71329, 71331, 71332).

Gutierrezia sarothrae (Pursh) Britton \& Rusby, 6814 TEX; 8492 TEX; 8620; 8656; 8823 BRIT; 8840, 8940; 8950 US; 8999; 9056 TEX, US; (NMC, MEXU, 8903). 
Gutierrezia sphaerocephala A. Gray, 5207 TEX; 6741 BRIT, TEX; 6777 TEX; 7832 BRIT, TEX; 8985 BRIT, TEX; 9032 TEX.

Gutierrezia sericocarpa (A. Gray) M.A. Lane, 8882 BRIT; 8890 US.

Gutierrezia wrightii A. Gray, 6438, 8617; 7648 US; 8518 TEX 9032.

Gymnosperma glutinosum (Spreng.) Less., 4667, 6762; 7678 BRIT; 8264; 8270 US; 8813, 9053, 4964.

Helianthus annuus L., 5793, 7892.

Helianthus laciniatus A. Gray, 5700 ANSM; 5892 TEX; 5896 TEX; 5928 TEX; 7887 TEX.

Helianthus petiolaris Nutt. var. petiolaris, (TEX 125848).

Heliopsis parvifolia A. Gray, 7612 ANSM, BRIT, TEX, US; (TEX 126000).

Heterosperma pinnatum Cav., 6064, 6094; 6116 ANSM; 6167 TEX; 7249 TEX; 7604 ANSM, BRIT; 7760 TEX; 8271 ANSM, US; 8310 ANSM, BRIT, TEX; 8776 TEX (NMC 52954).

Heterotheca subaxillaris (Lam.) Britton \& Rusby, 5618 TEX; 9043 TEX.

Heterotheca villosa (Pursh) Shinners, 2759.

Heterotheca viscida (A. Gray) Harms, 4610; 4650 ANSM; 4692; 7130 TEX.

Hieracium abscissum Less., 6628 TEX; 8415.

Hieracium carneum Greene, 9153.

Hymenothrix wislizeni A. Gray, 2626; 5513 US; 5819 ANSM; 7223, 7836 BRIT, TEX; 8233; 8982 ANSM, BRIT; 9076 NMC.

Hymenoxys odorata DC., 5681 ANSM, BRIT, TEX: 7220.

Hymenoxys scaposa (DC.) K.F. Parker var. scaposa, 5958 TEX; 6757 ANSM, 6800, 6843 BRIT; 6971; 7532 TEX.

Isocoma plurifolia (Torr. \& A. Gray) Greene, 5676 TEX; 5689 BRIT.

Isocoma tomentosa G.L. Nesom, 5661 TEX, US; 7910 BRIT, TEX, US; (NMC 58628).

Iva ambrosiaefolia (A. Gray) A. Gray, 4891 ANSM; 7835 BRIT, TEX; 8482 ANSM, TEX; 8989 US.

Iva dealbata A. Gray 8073; 8152 ANSM, BRIT, TEX.

Jefea brevifolia (A. Gray) Strother, 5807, 7806 US; 8581; 8708 BRIT; 8954 BRIT; 8992 ANSM, BRIT; 9070 NMC.

Lasianthaea podocephala (A. Gray) K.M. Becker, 5602 ANSM, BRIT, TEX; 7704 TEX.

Lygodesmia texana (Torr. \& A. Gray) Greene ex Small, 5081; 5345 TEX; 5386 ANSM, BRIT, US; 6817; 7078 ANSM; 8065; 8102 ANSM, BRIT.

Machaeranthera canescens A. Gray ssp. glabra (A. Gray) B.L. Turner, (TEX 137883).

Machaeranthera gracilis (Nutt.) Shinners, 8780 TEX; 8211 TEX; 7280 TEX.

Machaeranthera gypsitherma G.L. Nesom, Vorobik \& R.L. Hartm., (TEX 138006, 138007, 138008). 
Machaeranthera gypsophila B.L. Turner, 5897 TEX; 7572 TEX.

Machaeranthera parviflora A. Gray, (TEX 138131, 138137).

Machaeranthera pinnatifida (Hook.) Shinners var. chihuahuana B.L. Turner \& R.L. Hartm., 4828 ANSM, TEX; 4969 ANSM, TEX; 5033 TEX; 5666 TEX; 5748 TEX; 6940 TEX; 7130 TEX; 7140 ANSM, TEX, US; 7561 TEX; 6940 TEX; 8059 ANSM, TEX; 7183 ANSM, BRIT, TEX; 9020 BRIT, TEX; 9036 TEX; 9078 TEX.

Machaeranthera pinnatifida (Hook.) Shinners var. pinnatifida 4169a, 7183 ANSM, BRIT, TEX; 9020 BRIT, TEX; 9036 TEX; 9078 TEX (NMC 54842).

Machaeranthera riparia (Kunth) A. G. Jones, 8642 ANSM, TEX.

Machaeranthera tanacetifolia (Kunth) Nees, 7902 ANSM, TEX; 8116 ANSM, TEX, US; 8935 ANSM, BRIT, TEX, US.

Machaeranthera turneri M.L. Arnold \& R.C. Jacks. (TEX 138610, 138611, 138612)*

Melampodium leucanthum Torr. \& A. Gray var. leucanthum, 5988 TEX; 6209 TEX; 6718, 6771 BRIT, 6872 ANSM, BRIT, US; 6937 TEX; 7188 ANSM.

Melampodium strigosum Stuessy, 6148 ANSM, TEX; 6708 ANSM, TEX; 7343 TEX; 7664 TEX.

Nicolletia edwardsii A. Gray, 6975 ANSM.

Parthenium argentatum A. Gray, 5784, 5789; 6727 US.

Parthenium bipinnatifidum (Ortega) Rollins, 8843 ANSM, BRIT, TEX.

Parthenium confertum A. Gray, 2628; 4888 TEX; 5721 TEX; 6353, 6357; 6944 TEX; 7095

US; 7571 TEX; 8865 TEX, 9033 TEX; 5721.

Parthenium hysterophorus L., 6379 TEX; 7996 BRIT, TEX; 8843 TEX.

Parthenium incanum Kunth, 4881 TEX; 4962 TEX; 5334; 5721 TEX; 5803; 7899 BRIT, NMC, TEX; 8484 ANSM, TEX, US.

Pectis angustifolia Torr. var. angustifolia, 5720 TEX.

Pectis filipes Harv. \& A. Gray var. subnuda Fernald, 7938 TEX.

Pectis papossa Harv. \& A. Gray var. grandis D. J. Keil., 5675 ANSM, BRIT, TEX, US; 7901 BRIT, TEX.

Pectis prostrata Cav., 4674, 5139, 5148 BRIT; 5570; 5729 TEX; 5881, 6133, 6143, 6147

ANSM; 6465; 8041 ANSM; 8090; 8118.

Perityle microcephala A. Gray, 10771 MEXU, NMC, UC (NMC 66626).

Perymenium buphthalmoides DC., 4919 BRIT; 5555, 5562; 5600 ANSM, BRIT; 8283, 8301;

8430 BRIT; 8436; 8829 ANSM, NMC, 8970 US.

Perymenium mendezii DC. 4631.

Porophyllum linaria (Cav.) DC. *

Porophyllum scoparium A. Gray, (TEX 60596, 60598).

Psacalium decompositum (A. Gray) H. Rob. \& Brettell, 9563. 
Psilactis brevilingulata Sch. Bip. ex Hemsl., 8634 TEX; 9034 ANSM, TEX.

Psilostrophe tagetina (Nutt.) Greene, 4832; 5556 US; 5820 NMC, TEX; 6443 BRIT; 6455; 6717 BRIT; 6760 NMC; 7057 ANSM; 7207, 8163.

Pyrrhopappus multicaulis DC., 8649 ANSM, BRIT, TEX.

Ratibida latipalearis E.L. Richards *

Roldana hartwegii (Benth.) H. Rob. \& Brettell, 8739 ANSM, BRIT, TEX, US.

Sanvitalia abertii A. Gray, 5730 NMC; 5775 US; 5878, 6342; 6480 US; 6730 BRIT; 6782 ANSM; 7997 NMC; 8088 TEX.

Sanvitalia angustifolia Engelm. ex A. Gray, 8845 ANSM, TEX; 8856; (TEX 130886).

Sanvitalia procumbens Lam., 6376 TEX.

Sartwellia gypsophila A.M. Powell \& B.L. Turner, (TEX 86173, 86175).

Sartwellia puberula Rydb., 6955 ANSM; 8694 ANSM, BRIT, NMC, TEX, US; (TEX 86215).

Schkuhria multiflora Wedd., 8524 US; 8725 TEX; (TEX 61072).

Schkuhria pinnata (Lam.) Kuntze ex Tell. var. guatemalensis (Rydb.) McVaugh, 6208 TEX; (TEX 60918, 60923, 60931).

Schkuhria pinnata (Lam.) Kuntze ex Tell. var. virgata (La Llave) Heiser, (NMC 55767; TEX 60898).

Schkuhria pinnata (Lam.) Kuntze ex Tell. var. wislizeni (A. Gray) B.L. Turner, 6066 ANSM, US; 6079; 6104 ANSM; 6112, 6141, 6146, 6088, 6457, 6839; 8406 ANSM; 8849 ANSM.

Senecio flaccidus DC. var. douglasii (DC.) B.L. Turner \& T.M. Barkley, 7055 TEX; (TEX 191281, 136920).

Senecio flaccidus DC. var. flaccidus, 2469; 4164; 5267 ANSM, US, 5379 BRIT, 6486; 6769

ANSM; 8191 ANSM; (TEX 63210, 63211, 63216, 63217, 191448).

Senecio millelobatus Rydb., (TEX 306329).

Senecio neomexicanus A. Gray *

Senecio riddellii Torr \& A. Gray, (TEX 191275).

Senecio tampicanus DC., (TEX 306359).

Senecio salignus DC., (NMC 73377; TEX 63304, 63309, 63310).

Simsia amplexicaulis (Cav.) Pers., 8610 BRIT, TEX; 8714; 8878.

Simsia foetida (Cav.) S.F. Blake, 6479 ANSM, BRIT.

Simsia lagascaeformis DC., 5851 TEX; 6479 TEX; 8470 TEX; 8957 ANSM, TEX.

Solidago juliae G. L. Nesom, (TEX 85810).

Solidago velutina DC., (TEX 139017, 139018).

Solidago wrightii A. Gray, 8914 ANSM, TEX.

Sonchus asper (L.) Hill, 7111a ANSM, TEX; 7382; 7593 ANSM, BRIT, TEX.

Sonchus oleraceus L., 4162. 
Stevia amblyolepis (B. L. Rob.) B. L. Rob. var. amblyolepis 8711 TEX, US.

Stevia micrantha Lag., 8746 ANSM, TEX, US; (TEX 118451, 118462).

Stevia ovata Willd. var. ovata, (NMC 56549).

Stevia plummerae A. Gray var. plummerae, (TEX 118559).

Stevia purpurea Pers., 7625 ANSM, BRIT, TEX, US.

Stevia salicifolia Cav. var. nana A. Gray, 7346 US.

Stevia salicifolia Cav. var. salicifolia, 4652 ANSM; 7255 ANSM, BRIT, TEX, US; 8445

ANSM; (NMC 53569; TEX 119716, 119719).

Stevia serrata Cav. var. arguta B.L. Rob., 8714 ANSM, TEX; 8878 US.

Stevia serrata Cav. var. serrata, 6070 US; 6637 TEX; 8367 ANSM; 8720 ANSM, US; 8896

ANSM, US; (TEX 119310, 119312).

Stevia tephra B.L. Rob., (TEX 119499).

Stevia viscida Kunth, (TEX 118163, 118164, 118185, 118190, 118192).

Symphyotrichum expansum (Poepp. ex Spreng.) G.L. Nesom, 5643 TEX; 5707 ANSM,

TEX; 7718 TEX; 8232 BRIT, TEX; 8374 TEX.

Tagetes lucida Cav., 8665; 7285 ANSM.

Tagetes micrantha Cav., 8314 TEX; (TEX 61807, 61815).

Taraxacum officinale Wiggers, 7796 ANSM

Thelesperma longipes A. Gray, 7574 TEX; 8600; 8917 ANSM; (TEX 132339, 132342, 132362).

Thelesperma megapotamicum (Spreng.) Kuntze var. megapotamicum, 4168; 7008 ANSM, BRIT, TEX; 7236 ANSM, BRIT; (TEX 132408, 132409, 132413).

Thymophylla acerosa (DC.) Strother, 6770 TEX; 6913 ANSM, BRIT; 6968 TEX; (TEX 57885, 57890, 57895).

Thymophylla aurea (A. Gray) Greene, 5815 TEX; 6370 ANSM; 6347; 6797a; 9024 ANSM; (TEX 58037, 58046, 58050).

Thymophylla pentachaeta (DC.) B. L. Rob. var. hartwegii (A. Gray) Strother, 6444 ANSM; 6982 ANSM; 7003 TEX; (TEX 58476, 58478, 58479).

Thymophylla pentachaeta (DC.) B. L. Rob. var. pentachaeta, 2625, 4612; 4818 TEX; 4890; 5782 TEX; 6949; 7216 ANSM, TEX; (TEX 58517, 58518, 58519, 58520, 58528, 58530).

Thymophylla tenuifolia (Cass.) Rydb., 8049 TEX; (TEX 306225).

Tithonia brachypappa B. L. Rob., 8407 ANSM; 8419 BRIT; 8463 US; 8686 ANSM, 9047 NMC.

Tithonia tubaeformis (Jacq.) Cass., 6441 ANSM, BRIT, TEX.

Tridax bicolor A. Gray, 4629, 5544 ANSM, BRIT; 5613; 5850 TEX; 6191 TEX; (TEX 29180, 132756, 132757). 
Trixis californica Kellogg var. californica, 2605, 3795, 4632, 4895; 5865 BRIT; 7789, 7858; 8561 ANSM, 8977 US; 9051, 4895; (TEX 66698, 66701, 66703, 66707, 66754, 66756).

Verbesina chihuahuensis A. Gray, (TEX 133855, 133856, 133857).

Verbesina encelioides (Cav.) A. Gray var. encelioides, 4190; 4810 BRIT; 5044, 7103 US; (TEX 1339039).

Verbesina encelioides (Cav.) A. Gray var. exauriculata B.L. Rob. \& Greenm., (TEX 133968, 133975, 133976, 133977, 133978).

Verbesina longipes Hemsl., 5951 TEX; 133772).

Verbesina parviflora (Kunth) S.F. Blake, 7308 TEX; 7690 US; 9044 US; (TEX 134121, 182235).

Viguiera annua (M. E. Jones) S.F. Blake, 8651 ANSM; 8897 ANSM, US; 8965 ANSM.

Viguiera cordifolia A. Gray, 7324 ANSM, TEX, US; 7689 ANSM, TEX; 8605 ANSM; 8680 TEX, US; 8831 BRIT, TEX; 8964 BRIT, TEX, US; 9019 ANSM, BRIT; 9324 TEX.

Viguiera decurrens A. Gray, (NMC 44221; TEX 135310, 135311).

Viguiera dentata (Cav.) Spreng. var. dentata, 6224 TEX; 6281 TEX; 6570 TEX; 6719 BRIT, TEX; 7596 ANSM, TEX; 8687 BRIT, US; 8795 ANSM, BRIT; 8991 ANSM, BRIT, NMC, TEX, US; 9019 TEX; 9049 TEX; (TEX 135605, 135624, 135627).

Viguiera linearis (Cav.) Sch. Bip., 4694 ANSM; 8241 ANSM, TEX; 8566, 8569 ANSM; 8948 ANSM, BRIT, NMC, TEX; 8961 ANSM, BRIT, TEX.

Viguiera longifolia (B.L. Rob. \& Greenm.) S.F. Blake, 6339 ANSM; 6373 ANSM; 6860 US; 8763 US; 8813a ANSM.

Viguiera multiflora (Nutt.) S.F. Blake var. multiflora, 6595 TEX; 6620 TEX; 6847 ANSM, TEX; 8881 TEX; 8903a BRIT, TEX; 8965 BRIT, TEX; (TEX 136092, 136093, 136096).

Viguiera stenoloba S.F. Blake, 8948 NMC; (TEX 135908, 135909, 135912).

Viguiera triloba (A. Gray) Olsen, 7734; (TEX 135962, 135963, 135966).

Xanthium strumarium L., 5850 BRIT, TEX; 9018.

Xanthocephalum gymnospermoides (A. Gray ex Rothr.) Benth. \& Hook., 7310 TEX, US; 8375 TEX; 8617 TEX; 8653; 7722 TEX; 8376 TEX; 8886 TEX.

Zaluzania discoidea A. Gray, 7353 ANSM; 7669 TEX.

Zinnia acerosa (DC.) A. Gray *

Zinnia grandiflora Nutt., 4155, 4894, 5020; 6446 BRIT; 6748 US; 7031; 7217 ANSM; 7872 BRIT; 8119 ANSM.

Zinnia peruviana (L.) L., 4648, 4675; 5542 BRIT; 6105, 6171, 7634; 7932 ANSM, BRIT. Zinnia violacea Cav., (TEX 55387). 
Zinnia zinnioides (Kunth) Olorode \& A. M. Torres, (TEX 55440).

\section{BEGONIACEAE}

Begonia gracilis Kunth, 8546a BRIT; 8765 ANSM, BRIT.

\section{BERBERIDACEAE}

Berberis trifoliolata Moric., 5787, 6958.

\section{BIGNONIACEAE}

Chilopsis linearis (Cav.) Sweet, 5149 BRIT; 7221 ANSM, BRIT; 8287 ANSM.

Tecoma stans (L.) Juss. ex Kunth, 2621; 4856 ANSM, BRIT; 7298; (NMC 52808).

\section{BORAGINACEAE}

Antiphytum floribundum (Torr.) A. Gray, 5428 BRIT; 5440 ANSM, NMC, TEX; 6807; 8793 ANSM; 8450 BRIT.

Antiphytum heliotropioides A. DC., 7785 ANSM, BRIT, TEX.

Cordia parvifolia A. DC. *

Cryptantha albida (Kunth) I.M. Johnst., (TEX 224748, 224753, 224754).

Cryptantha mexicana (Brandegee) I. M. Johnst., (TEX 224903, 224905).

Cryptantha pusilla (Torr. \& A. Gray) Greene 4185; (TEX 224970).

Heliotropium convolvulaceum (Nutt.) A. Gray, (TEX 225317, 225320, 225321).

Heliotropium curassavicum L. var. curassavicum, 5663; 7917 ANSM, BRIT, 8978, 9735.

Heliotropium glabriusculum (Torr. ) A. Gray *

Heliotropium greggii Torr., 5630, 5667; 5817 BRIT; 6351 ANSM; 6369; 7898 BRIT, NMC; 9013.

Heliotropium molle (Torr. ) I. M. Johnst. *

Heliotropium procumbens Mill., 6487 ANSM, BRIT, TEX.

Lappula occidentalis (S. Watson) Greene *

Lithospermum cobrense Greene, 6215 ANSM, TEX; (NMC 52834; TEX 225864, 225853).

Lithospermum incisum Lehm., 7369 ANSM, BRIT, TEX.

Lithospermum multiforum A. Gray, 7657 ANSM, TEX; (TEX 225977).

Tiquilia canescens (DC.) A. T. Richardson var. canescens, 5655; 5659 TEX; 6922 ANSM; (TEX 223136, 223137, 223141, 223142).

Tiquilia gossypina (Wooton \& Standl.) A.T. Richardson, (TEX 223309, 223310, 223312, 223313).

Tiquilia greggii (Torr \& A. Gray) A. T. Richardson, 5745 ANSM, BRIT; 6724 TEX; 8495, 8709 ANSM; 7898 ANSM, TEX; 8978 TEX; 9013 TEX. 


\section{BRASSICACEAE}

Brassica campestris L., 2761 ANSM; 6905.

Descurainia pinnata (Walt.) Britton var. pinnata, 4153; 6883 BRIT, TEX; 6899 ANSM, BRIT.

Draba cuneifolia Nutt. ex Torr. \& A. Gray *

Eruca sativa Mill., 2756; 5821 BRIT; 6904 BRIT.

Halimolobos diffusus (A. Gray) O. E. Schultz., 7954a TEX.

Lepidium alyssoides A. Gray var. angustifolium (C.L. Hitchc.) Rollins, (TEX 148259).

Lepidium austrinum Small, (TEX 148209).

Lepidium montanum Nutt., 4189.

Lepidium sordidum A. Gray, 7257 TEX; 7294; 7761 ANSM, BRIT; (TEX 148297).

Lepidium virginicum L., 6710; 6901 TEX; 6909 ANSM.

Lesquerella fendleri (A. Gray) S. Watson, 4614 ANSM; 4930 TEX; 4935 ANSM; 7146.

Lesquerella purpurea (A. Gray) S. Watson, 5077 ANSM.

Mancoa pubens (A. Gray) Rollins, 4149a.

Nerisyrenia camporum (A. Gray) Greene, 5684, 5686, 6969; 6973 ANSM.

Nerisyrenia gypsophila J. D. Bacon, (TEX 148761, 148860, 148861, 148863).

Pennellia longifolia (Benth) Rollins, 7693 ANSM, BRIT; 8342 BRIT; 8555 BRIT.

Pennellia micrantha (A. Gray) Nieuwl. *

Rorippa nasturtium-aquaticum (L.) Hayek, 6885 ANSM, BRIT, TEX; (TEX 149095).

Scoliaxon mexicanus (S. Watson) Payson *

Sisymbrium auriculatum A. Gray, (TEX 149207).

Sisymbrium irio L., 2755 ANSM; 7159 ANSM, TEX.

Sisymbrium linearifolium (A. Gray) Payson, 7676 TEX

Streptanthus carinatus Wright. ex A. Gray, 8413 ANSM. *

Synthlipsis greggii A. Gray, 6935 ANSM, BRIT.

Thelypodium wrightii (A. Gray) Rydb., 7621 ANSM; 7949 ANSM, BRIT, TEX; 8020 BRIT,

NMC.

\section{CACTACEAE}

Coryphantha echinus (Engelm.) Britton \& Rose, 7024 UAT.

Coryphantha macromeris (Engelm.) Lem., 7122B UAT; 7809a UAT; 7867a; 8852a UAT; 8852d UAT; (TEX 181845, 181847).

Coryphantha poselgeriana (Dietr.) Britton \& Rose, 7025 UAT.

Coryphantha ramillosa Cutak, 7847a UAT.

Echinocactus setispinus Engelm., 6686.

Echinocactus parryi Engelm. * 
Echinocereus dasyacanthus Engelm., 7021 UAT; 7581 UAT; 7027 UAT; 8790d.

Echinocereus palmeri Britton \& Rose *

Echinocereus pectinatus (Scheidw.) Engelm., 6678, 6680, 6681, 6682; 6333a; 6683.

Echinocereus stramineus (Engelm.) Engelm. ex F. Seitz *

Echinocereus triglochidiatus Engelm. var. arizonicus (Rose ex Orcutt) Benson, 9160 UAT.

Echinocereus triglochidiatus Engelm. var. neomexicanus (Standl.) W. T. Marshall, 8302a UAT.

Echinomastus intertextus (Engelm.) Britton \& Rose 7587 UAT; 8231a UAT.

Escobaria chihuahuensis Britton \& Rose ssp. chihuahensis, 8495a UAT.

Escobaria dasyacantha (Engelm.) Britton \& Rose, 7028.

Escobaria vivipara (Nutt.) Haw., 7023.

Grusonia grahamii (Engelm.) H. Rob. *

Mammillaria heyderi Muehl. var. meiacantha (Engelm.) L.D. Benson, 7580 UAT; 8709 UAT; 8302b.

Mammillaria pottsii Scheer ex Salm-Dyck, 7023; 7589 UAT.

Mammillaria wrightii Engelm. *

Opuntia azurea Rose *

Opuntia engelmannii Salm-Dyck, 8267a.

Opuntia imbricata (Haw.) DC. (obs. pers.).

Opuntia macrocentra Engelm. *

Opuntia leptocaulis DC., 8483.

Opuntia lindheimeri Engelm., (TEX 271251).

Opuntia macrocentra Engelm., (TEX 271259).

Opuntia phaeacanhta Engelm., 6687.

Opuntia rufida Engelm. *

Sclerocactus uncinatus Britton \& Rose, 7835 UAT.

Thelocactus bicolor (Galeotti ex Pfeiffer) Britton \& Rose, 8852a UAT.

\section{CAMPANULACEAE}

Lobelia fenestralis Cav., 8357 BRIT; 8402 ANSM; 8547 ANSM, BRIT; 8729 BRIT; 8751 BRIT; (TEX 80208, 80209).

\section{CAPPARIDACEAE}

Cleomella longipes Torr., 4208 ANSM; 5680 ANSM, BRIT; 7908 ANSM; 8639 ANSM, BRIT.

Polanisia dodecandra (L.) DC. var. trachysperma (Torr. \& A. Gray) H. H. Iltis, 4916 TEX; 5011, 5103, 5111 BRIT; 5184 ANSM; 8177, 8790. 
Polanisia uniglandulosa (Cav.) DC., 3433a ANSM; 9410.

CAPRIFOLIACEAE

Lonicera pilosa (Kunth) Willd., 7169a BRIT, TEX.

\section{CARYOPHYLLACEAE}

Arenaria ludens Shinners, 8384 ANSM TEX.

Cerastium brachypodum (Engelm. ex A. Gray) B. L. Rob. ex Britton *

Corrigiola andina Triana \& Planch. *

Drymaria arenarioides (Benth.) Willd. ex Roem. \& Schult, 4163; 4897 BRIT; 4992; 5019

ANSM; 5256, 5071; 5254 BRIT; 5401, 5404; 7002 BRIT, 7222, 7756 ANSM, BRIT; 7935

NMC; 8148; 8907 NMC; 8944 BRIT; (NMC 48783, 48792, 52453, 58458).

Drymaria glandulosa C. Presl *

Drymaria laxiflora Benth., 7740 ANSM, BRIT; 8133 MEXU, NMC, RM; 8736 BRIT; (TEX 239343, 239346, 239348).

Drymaria leptophylla (Cham. \& Schltdl.) Rohrb. var. nodosa (Engelm.) J.A. Duke, 8517

BRIT; 8395 ANSM; (TEX 239310).

Drymaria lyropetala I. M. Johnst. var. lyropetala *

Drymaria molluginea (Lag.) Didr., 4637.

Drymaria pachyphylla Woot. \& Standl., 7358 ANSM.

Paronychia wilkinsonii S. Watson, (NMC 48796).

Silene laciniata Cav., 7707 ANSM; 8366 ANSM; 9135.

Silene scouleri Hook. var. pringlei (S. Watson) Hitchc., 7607a; 8029 ANSM, BRIT, NMC.

Stellaria prostrata Baldwin, 7989 ANSM; 8000 ANSM, BRIT, TEX.

Stellaria umbellata Turcz., 5304, 5391; 5419 ANSM, BRIT; 5801; 6842 ANSM, BRIT.

\section{CHENOPODIACEAE}

Allenrolfea occidentalis (S. Watson) Kuntze *

Atriplex acanthocarpa (Torr.) S. Watson ssp. acanthocarpa, 5734 ANSM, BRIT, TEX;

(TEX 172698, 172700, 172704).

Atriplex canescens (Pursh) Nutt., 5891 ANSM; (TEX 172785, 172797).

Atriplex obovata Moq. (TEX 172910, 172915, 172933).

Atriplex rosea L. *

Chenopodium albescens Small, 7293 TEX; 7312 ANSM, TEX, 7318 ANSM, TEX.

Chenopodium album L. 7295a TEX; 7769 ANSM, BRIT.

Chenopodium berlandieri var. sinuatum (Muhl.) Wahl, (TEX 173070, 173092). 
Chenopodium graveloens Willd., 8274 ANSM, BRIT; (TEX 173129, 173130).

Chenopodium incanum (S. Watson) Heller, 7181 TEX; 7238 ANSM, TEX; (TEX 173177).

Kochia scoparia (L.) Roth ex Schrad., 4192 ANSM; 6395 ANSM; 8131 TEX.

Salsola tragus L., 4904, 7886.

Suaeda suffrutescens S. Watson var. detonsa I.M. Johnst. 7907 ANSM, BRIT, TEX.

\section{CISTACEAE}

Helianthemum pringlei S. Watson, 7713a ANSM, BRIT; (TEX 159359).

\section{COCHLOSPERMACEAE}

Amoureuxia malvifolia A. Gray *

\section{CONVOLVULACEAE}

Convolvulus equitans Benth., 5202, 5495; 7060 ANSM, BRIT; 6964 BRIT.

Dichondra argentea Willd., 5470a, 6461, 7066, 7139a; 7920 BRIT; 8194; (NMC 48794).

Dichondra brachypoda Wooton \& Standl., 9154.

Evolvulus alsinoides L. var. angustifolius Torr., 4985 BRIT; 5340 ANSM; 5374, 7774a, 9074; (NMC 52821).

Evolvulus alsinoides L. var. hirticaulis Torr., 5546, 5864; 7032 BRIT; 7845 BRIT, 8552.

Evolvulus sericeus Benth., 7009, 7070; 7147 ANSM; 8139 ANSM.

Ipomoea batatas (L.) Lam. var. apiculata (M. Martens \& Galeotti) J. A. McDonald \& D. F.

Austin, 5592 ANSM, TEX; 5611 BRIT.

Ipomoea capillacea (Kunth) G. Don, 5431 TEX; 7693 ANSM, BRIT; 8184; 8312 BRIT.

Ipomoea cardiophylla A. Gray, 6445 ANSM, BRIT, TEX.

Ipomoea costellata Torr., 5855 ANSM, BRIT, TEX; (NMC 48788; TEX 242346).

Ipomoea hederacea (L.) Jacq., 5827 ANSM, BRIT, TEX; 5836, 8273 ANSM.

Ipomoea lindheimeri A. Gray, (TEX 242733).

Ipomoea madrensis S. Watson, 8325 ANSM, BRIT, TEX; 8333.

Ipomoea petrophila House, (TEX 242995).

Ipomoea pubescens Lam., 6120 NMC; 8258 ANSM; 8272 ANSM; 8428 BRIT; (TEX 217047).

Ipomoea purpurea (L.) Roth, (TEX 217071, 217099).

Ipomoea wrightii A. Gray, 6458 ANSM; 6483.

\section{CORNACEAE}

Cornus stolonifera Michx. * 


\section{CRASSULACEAE}

Echeveria mucronata Schltdl. *

Echeveria paniculata A. Gray, 7263 ANSM, TEX; 7729 NMC; 8021 ANSM, BRIT, TEX.

Echeveria strictiflora A. Gray, (TEX 168243).

Sedum greggii Hemsl., 8323 ANSM; 8358 ANSM, BRIT, TEX.

Sedum pringlei S. Watson *

Sedum vinicolor $\mathrm{S}$. Watson, (TEX 168650).

Sedum wrigthii A. Gray, 8740 ANSM, BRIT, TEX.

Villadia squamulosa (S. Watson) Rose *

\section{CUCURBITACEAE}

Apodanthera undulata A. Gray, 5337; 5361 BRIT; 5381 NMC; 5621 NMC; 7206 ANSM, BRIT; 7881 BRIT.

Cucurbita foetidissima Kunth, 5514, 7314 BRIT; 7088.

Cyclanthera dissecta (Torr. \& A. Gray) Arn., 9422; (TEX 79125) .

Cyclanthera ribiflora (Schltdl.) Cogn., 8427 BRIT.

\section{CUSCUTACEAE}

Cuscuta applanata Engelm., (TEX 155594).

Cuscuta mitriformis Engelm. *

Cuscuta pentagona Engelm., (TEX 155730).

Cuscuta umbellata Kunth var. reflexa (Coult.) Yunck., 5510 ANSM, BRIT; (TEX 155792).

\section{ERICACEAE}

Arbutus xalapensis Kunth, 8408 BRIT; 8588 ANSM.

Arctostaphylos pungens Kunth, 2737 ANSM; 7363 ANSM.

\section{EUPHORBIACEAE}

Acalypha neomexicana Muell. Arg., 4923 BRIT, TEX; 4996.

Acalypha ostryaefolia Riddell, 4657 ANSM.

Acalypha phleoides Cav., 7311 ANSM; 7319 ANSM, BRIT; (TEX 215074).

Bernardia obovata I.M. Johnst., 8869a.

Croton fruticulosus Torr., (TEX 92519).

Croton pottsii (Klotszch) Müll. Arg. var. pottsii, 6113, 6121, 6798, 7411b; (NMC 58784).

Croton pottsii (Klotszch) Müll. Arg. var. termophilus (I. M. Johnst.) I. M. Johnst., 4911, 5123; 6291 ANSM 7004. 
Croton sancti-lazari Croizat, (TEX 93103, 93105, 93106, 93107, 93108).

Croton suaveolens Torr., (TEX 93151).

Croton texensis (Klotszch) Müll. Arg., (TEX 93192).

Ditaxis neomexicana (Müll. Arg.) A. Heller, 6867 ANSM, TEX; 6738 BRIT.

Euphorbia albomarginata Torr. \& A. Gray, 7073 ANSM; 7889.

Euphorbia antisyphylitica Zucc., 7018 BRIT.

Euphorbia anychioides Boiss., 7104 TEX.

Euphorbia arizonica Engelm., 7865 TEX.

Euphorbia bilobata Engelm., 8855 TEX.

Euphorbia cuphosperma (Engelm.) Boiss., (TEX 94125).

Euphorbia davidii Subils, (TEX 94227).

Euphorbia dentata Michx., 6737a ANSM.

Euphorbia exstipulata var. lata Warnock \& M.C. Johnst., 5132; 5147 TEX; 7850 TEX.

Euphorbia glyptoseprma Engelm., 4853 BRIT; 5104 ANSM; 5107 ANSM; 5178; 5189; 8060; 8144.

Euphorbia heterophylla L., 4638, 4690; 5265 ANSM, BRIT, TEX; 5849 BRIT; 7945 ANSM, BRIT; 9016 ANSM, BRIT, TEX.

Euphorbia hyssopifolia L., 4623, 5005 ANSM; 5097 TEX; 5477; 7862; 7882 BRIT; 8094; 8121.

Euphorbia indivisa (Engelm.) Tidestr., 6751 TEX; 8567 TEX.

Euphorbia macropus (Klotzsch \& Garcke) Boiss., 7705 TEX.

Euphorbia micromera Boiss., 2620.

Euphorbia radians Benth., 4177, 7012; 7085 BRIT.

Euphorbia serpens Kunth, 8064; 8093 TEX.

Euphorbia serpyllifolia Pers., 4976 ANSM; 5107a ANSM; 5109; 7705; 7828a BRIT; 8252 ANSM

Euphorbia serrula Engelm., 4620.

Euphorbia stictospora Engelm., 5497 TEX; 5500, 5527, 6356, 6366; 6742 ANSM; 8979, 5518.

Euphorbia villifera Scheele, 5116 ANSM, TEX; 5210, 5228; 7339 TEX; 7814, 8077, 8646.

Jatropha dioica Cerv. var. dioica, 5653, 6923.

Jatropha dioica var. graminea McVaugh, 6960, 7876.

Jatropha macrorhiza Benth., 5363 ANSM, NMC; 7177 ANSM; 7878 BRIT.

Phyllanthus polygonoides Spreng., 4951 BRIT; 4986 BRIT; 5326 ANSM; 8867 BRIT.

Tragia amblyodonta (Müll. Arg.) Pax \& K. Hoffm., (TEX 102810).

Tragia laciniata (Torr.) Müll-Arg., 5033; 5035 TEX; 7654 TEX; (TEX 102884, 102890). 


\section{FAGACEAE}

Quercus arizonica Sarg., 8277 ANSM, NMC; 8513 NMC; 8873 ANSM, NMC; (NMC 52815).

Quercus arizonica Sarg. x Q. grisea Liebm., (NMC 68907).

Quercus x basaseachensis C. H. Mull. pro sp., 8335 BRIT, NMC.

Quercus chihuahuensis Trel., 4630, 4945; 4953 ANSM; BRIT, NMC; 5554; 6841 NMC;

7199 NMC; 7958 BRIT; 8825 ANSM, BRIT, NMC; (NMC 56884, 62841).

Quercus coccolobifolia Trel., 8583 ANSM, BRIT, NMC; 8589 ANSM, BRIT, NMC.

Quercus deliquescens C.H. Mull. (TEX 169353, 235480).

Quercus depressipes Trel., 2741; 7361 BRIT, NMC; 7732 BRIT, NMC; 8309 BRIT, NMC; 8343 BRIT, NMC; 8710 BRIT, NMC.

Quercus emoryi Torr., 2742, 2743; 2744 ANSM; 4688, 5269, 6164; 6990 ANSM; 7197 ANSM, BRIT; 8004 NMC; 8587 ANSM, BRIT, NMC; 8590 ANSM; 8719 ANSM, BRIT, NMC; 8898 NMC; (NMC 52791, 52824, 57231, 68996).

Quercus grisea Liebm., 2740; 5270 NMC; 6691 BRIT, 7198 BRIT, NMC; 8242; 8339 ANSM; 8446 BRIT, 8466 BRIT, NMC; 8591; 8602 ANSM, TEX; 8613 BRIT; 8820 BRIT, NMC.

Quercus hypoleucoides A. Camus, 2739; 6998 ANSM; 8338 ANSM, BRIT, NMC; 8584 NMC.

Quercus oblongifolia Torr. *

Quercus pungens Liebm. var. pungens 8858 ANSM, BRIT, NMC; (TEX 241846).

Quercus rugosa Née, 7259; 7364 BRIT, NMC; 8337 ANSM, NMC; 8744 BRIT; 8670 ANSM,

NMC; 8764 BRIT, NMC; 8773 NMC; 8879 ANSM, NMC; (TEX 235014).

Quercus viminea Trel., (TEX 235298).

FOUQUIERACEAE

Fouquieria splendens Engelm., 2470; 8836b.

FUMARIACEAE

Corydalis aurea Willd. var. occidentalis Engelm., 6888 ANSM; (NMC 52566).

\section{GARRYACEAE}

Garrya ovata Benth., 9424; (TEX 163992).

Garrya wrightii Torr., 8016 ANSM, TEX; (TEX 154053).

\section{GENTIANACAEAE}

Centaurium calycosum Fernald var. calycosum, 8637 ANSM; (TEX 251193).

Centaurium nudicaule B. L. Rob., (NMC 45862; TEX 251267, 251268, 251270). 
Eustoma exaltatum (L.) Griseb., 5637 ANSM; 5652 BRIT; 7912 ANSM, TEX.

Gentianella wislizeni (Engelm.) J. M. Gillett, 8903 ANSM, BRIT, TEX.

Halenia recurva (Sm.) Allen, 8909 TEX.

GERANIACEAE

Erodium cicutarium (L.) L'Hér. ex Aiton *

Geranium gracile Engelm., 8364 ANSM, BRIT.

Geranium caespitosum E. James, 8501 ANSM; (TEX 154664, 154667).

Geranium wislizeni S. Watson, 8731 NMC; 8758 ANSM, BRIT.

HYDRANGEACEAE

Fendlerella utahensis (S. Watson) A. Heller *

Philadelphus bifidus (C. L. Hitchc.) S. Y. Hu, 8344 ANSM, BRIT, TEX; (TEX 154338).

Philadelphus microphyllus A. Gray

Philadelphus occidentalis A. Nelson*

HYDROPHYLLACEAE

Nama carnosum (Wooton) C.L. Hitchc., (TEX 248145).

Nama dichotomum (Ruiz \& Pav.) Choisy, 7211 ANSM, BRIT; (TEX 248183).

Nama havardii A. Gray, (TEX 248234, 248241).

Nama hispidum A. Gray, 2752 ANSM; 4883, 5159; 6893 ANSM; 7099 ANSM; (NMC 52840;

TEX 248303, 248311, 248312, 248313).

Nama stenophyllum A. Gray, (TEX 248587).

Nama undulatum Kunth, 5508 TEX; (NMC 52567; TEX 248636, 248643).

Phacelia coerulea Greene, (TEX 248683, 248684).

Phacelia congesta Hook., 7991 ANSM, BRIT, TEX.

Phacelia gypsogenia I.M. Johnst., (TEX 248757).

Phacelia infundibuliformis Torr., (TEX 248790, 248792).

Phacelia patuliflora (Engelm. \& A. Gray) A. Gray *

Phacelia robusta (J. F. Macbr.) I.M. Johnst., (TEX 248958, 248964, 248965).

Phacelia rupestris Greene, (TEX 248975).

HYPERICACEAE

Hypericum pauciflorum Kunth

JUGLANDACEAE

Carya wrightii Torr., 7982 ANSM, BRIT, TEX. 
Juglans major (Torr.) Heller, 6984 TEX; 7244 ANSM, BRIT, TEX; (TEX 171387).

Juglans microcarpa Berl. var. stewartii (I.M. Johnst.) W.E. Manning, (TEX 171409, 171410, 171411).

KOEBERLINIACEAE

Koeberlinia spinosa Zucc., 5688 ANSM.

\section{KRAMERIACEAE}

Krameria erecta Willd., (TEX 215888).

Krameria grayi Rose \& Painter, 6640 BRIT; 6972 ANSM; 7822 BRIT; 8990 TEX; 8494; 8497 BRIT; 9012 NMC; (TEX 215798, 215802, 215811).

Krameria lanceolata Torr., 4607 ANSM; 5599 BRIT; (TEX 189756).

Krameria paucifolia (Rose) Rose, 6910 ANSM, BRIT, TEX.

Krameria secundiflora Moc. \& Sessé ex DC., 4909 ANSM, BRIT; 4987 BRIT; 5015; (TEX 215610, 215614, 215615).

\section{LAMIACEAE}

Agastache pallida (Lindl.) Cory var. coriacea R.W. Sanders, 7710; 8352 ANSM; 8511 BRIT; 8910.

Agastache pallida (Lindl.) Cory var. pallida, (TEX 164076).

Hedeoma drummnondii Benth. *

Hedeoma nanum Greene, (TEX 164436, 164446).

Hedeoma plicata (Torr.) Briq., (TEX 164497).

Marrubium vulgare L., 4159 ANSM.

Monarda citriodora Lag. var. austromontana (Epling) B.L. Turner, 8509 ANSM; 8786 ANSM.

Salvia ballotaeflora Benth., 7974 ANSM, BRIT, TEX; (TEX 244355).

Salvia coccinea Juss., 7966 ANSM, BRIT, TEX; 8457 ANSM.

Salvia elegans Vahl, 8360 TEX.

Salvia emaciata Epling *

Salvia greggii A. Gray, (TEX 234684, 234685, 234686, 306929).

Salvia lycioides A. Gray, (TEX 234644, 234645).

Salvia macellaria Epling. *

Salvia microphylla Kunth, (TEX 249799, 249805, 249812).

Salvia pinguifolia (Fernald) Wooton \& Standl., 8426 ANSM, BRIT; (TEX 244348, 244351).

Salvia prunelloides Kunth, 8880 ANSM, TEX. 
Salvia reflexa Hornem., 8838 BRIT, TEX; 8925 NMC; (TEX 234080).

Salvia reptans Jacq., 5468, 5588, 5603; 6618 TEX; 8185 ANSM; 8500 BRIT.

Salvia roemeriana Scheele, (TEX 223962).

Salvia subincisa Benth., (TEX 244528, 244534).

Salvia tiliaefolia Vahl, 6103 NMC; 6125 ANSM; 6176 BRIT; 6706, 8238 ANSM; 8386 ANSM, BRIT.

Scutellaria microphylla Benth., 8003 BRIT, TEX; 8291 ANSM, BRIT, 8418 ANSM.

Scutellaria potosina Brandegee, 8291 BRIT.

Scuttellaria tessellata Epling, (NMC 52861).

Stachys coccinea Jacq., 7619 ANSM, BRIT, TEX; (TEX 250603, 250606).

Teucrium cubense Jacq., 6914; 7990 ANSM, BRIT.

\section{LEGUMINOSAE (CAESALPINIOIDEAE)}

Caesalpinia drummondii (Torr. \& A. Gray) Fisher, (TEX 191930).

Caesalpinia gilliesii (Hook.) D. Dietr., 5685 BRIT; 4178; (TEX 250246).

Chamaecrista leptadenia Greenm., (TEX 97313, 97317).

Chamaecrista nictitans (L.) Moench ssp. nictitans var. mensalis (Greenm.) Irwin \& Barneby, 4621 ANSM; 4672 ANSM; 5610, 5852 BRIT; 6072, 6095; 6846, 8073, 8126; 8143 MEXU; 8178.

Hoffmannseggia glauca (Ortega) Eifert, 4171, 4702, 5691, 5816, 5824, 6352, 6355, 6946;

7231 TEX; 8042 ANSM; 8067 BRIT; 8120 BRIT; 9028; (TEX 191965).

Parkinsonia aculeata L., 7111, 5623.

Pomaria jamesii (Torr. \& A. Gray) Walp., 7184 BRIT, TEX; (TEX 97603).

Pomaria multijuga (S. Watson) B. B. Simpson, (TEX 97646).

Senna bauhinioides (A. Gray) H. S. Irwin \& Barneby, 2614, 4669; 4798 BRIT; 7100 MEXU;

7235 BRIT, MEXU; 7928; 8106 ANSM, BRIT, TEX; 8154 MEXU; 8995 BRIT; (TEX 97761, 97765).

Senna lindheimeriana (Scheele) H. S. Irwin \& Barneby, 5742 BRIT, MEXU, NMC.

Senna pilosior (Macbr.) H. S. Irwin \& Barneby, 7802 ANSM, BRIT, MEXU; NMC, TEX;

7823, 9011a, 9083 BRIT.

Senna ripleyana (Irwin \& Barneby) H. S. Irwin \& Barneby, 5772, 5785.

Senna wislizeni (A. Gray) H. S. Irwin \& Barneby var. wislizeni, 2617; 5001 BRIT; 5664

NMC; 6792 MEXU; 8952 BRIT; 9062 NMC; (TEX 247258).

\section{LEGUMINOSAE (LOTOIDEAE)}

Aeschynomene fascicularis Schltdl. \& Cham., 5588a BRIT.

Astragalus allochorus Gray var. playanus (Jones) Isely, 6897 BRIT, NMC. 
Astragalus austrinus (Small) E.D. Schulz var. austrinus, (TEX 260719).

Astragalus emoryanus (Rydb. ) Cory, (TEX 260771).

Astragalus lentiginosus Hook. *

Astragalus mollissimus Torr. var. earlei (Greene ex Rydb.) Tidestr., 4146 BRIT; 4179, 6763; 6890 ANSM, MEXU; (TEX 260958).

Astragalus mollissimus Torr. var. irolanus (M.E. Jones) Barneby, 2485, 4148; 5047; 6890 BRIT, TEX.

Astragalus nuttallianus A. DC. var. austrinus (Small) Barneby, 6889 ANSM, BRIT, NMC, TEX; 6896 MEXU.

Astragalus pringlei S. Watson, 2484; 6908 BRIT, TEX; 7069 TEX.

Astragalus quinqueflorus S. Watson, 6891 ANSM, BRIT, NMC, TEX; 7084.

Astragalus thurberi A. Gray *

Astragalus vaccarum A. Gray, (TEX 260614, 260615, 260616, 260617).

Astragalus wootonii Sheld., 2607, 4186; 6897 NMC.

Brongniartia minutifolia S. Watson*

Cologania angustifolia Kunth, 7170; 7279 BRIT; 7683 MEXU; 9136; (NMC 52571).

Cologania obovata Schltdl., 7712 MEXU; 8316 MEXU.

Coursetia caribaea (Jacq.) Lavin var. caribaea 4649, 6850 NMC; 6866 BRIT, MEXU, TEX.

Coursetia caribaea (Jacq.) Lavin var. sericea (A. Gray) Lavin, 7860 ANSM, BRIT, MEXU, TEX; 8236 BRIT; (NMC 53527; TEX 220211, 220213, 220215).

Coursetia glabella (A. Gray) Lavin, 9151 MEXU.

Crotalaria pumila Ortega, 4640, 4691, 5209; 6090 BRIT; 6096 NMC; 6107 NMC; 6436; 6440 MEXU; 6739 MEXU, NMC; 8036 BRIT; 8107; 9017 ANSM, BRIT, NMC; 9042 BRIT, TEX.

Dalea albiflora A. Gray, (TEX 247315, 247319, 247320).

Dalea bicolor Humb. \& Bonpl. ex Willd. var. argyraea (A. Gray) Barneby, (TEX 247346, 247348).

Dalea brachystachys A. Gray, 5719 BRIT; 6420 ANSM; 6437 TEX; 6450 BRIT; 6478 MEXU; 6608 BRIT, 6714, 6722 BRIT; 6789 MEXU; 6828, 8842, 8924; (TEX 247482).

Dalea filiformis A. Gray, 8506 BRIT.

Dalea foliolosa (Aiton) Barneby var. foliolosa, 6067; 6080 BRIT; 6114; 6123 ANSM; 6127 BRIT; 8373 ANSM, BRIT, MEXU; 8508; 8775 BRIT.

Dalea formosa Torr., 6826 NMC; 6916 ANSM, BRIT; 6974 MEXU.

Dalea grayi (Vail) L.O. Williams, 8347 ANSM, BRIT; 8441 BRIT; 8654 BRIT.

Dalea humilis G. Don, 4698. 
Dalea jamesii (Torr.) Torr. \& A. Gray, 8098 TEX.

Dalea lachnostachya A. Gray, 5128 BRIT, NMC; 6715 BRIT, MEXU; 6827 BRIT, 6789, 6827 MEXU; 7191 BRIT; 7212 BRIT, NMC; TEX; 7843 BRIT.

Dalea leporina (Aiton) Bullock, 8631 MEXU; 8757 BRIT, MEXU, NMC; (TEX 221971, 221973).

Dalea leucostachya A. Gray var. leucostachya, 7662 BRIT, TEX; 8248 BRIT.

Dalea lutea (Cav.) Willd. var. lutea, 6986 BRIT; 8459 ANSM, BRIT, MEXU; (TEX 214068).

Dalea mollis Benth., 6829 TEX.

Dalea nana Torr. ex A. Gray var. carnescens (Rydb.) Kearney \& Peebles, 5138; 5143 BRIT; 5327 MEXU; 5548 ANSM; 6451, 6774 BRIT; 6820, 6844 TEX; 6848 BRIT; 6857 BRIT; 6967 ANSM; 7083 ANSM, MEXU; 7838 BRIT, 8288; 8554 BRIT, MEXU.

Dalea neomexicana (A. Gray) Cory var. neomexicana, 6964 BRIT; 8476 BRIT.

Dalea pogonathera A. Gray var. pogonathera 5121 BRIT, MEXU; 5221, 6705; 6716 BRIT, MEXU; 6753 BRIT; 6778 NMC; 6938 MEXU; 7059; 7133 BRIT; 7844 BRIT, 8114 MEXU.

Dalea prostrata Ortega, 6845 TEX; 6758.

Dalea simulatrix Barneby *

Dalea versicolor var. canescens (Rydb.) Barneby, 8259.

Dalea versicolor var. glabrescens (Rydb.) Barneby, 8603; 8912 MEXU.

Dalea versicolor var. sessilis (A. Gray) Barneby, 8596 BRIT.

Dalea viridiflora $\mathrm{S}$. Watson, 8608 BRIT, MEXU, NMC.

Dalea wrightii A. Gray, 4978; 4993 BRIT; 5074 BRIT; 5282, 5319; 5330 ANSM; 7837 BRIT; 7869.

Desmodium angustifolium (Kunth) DC., 7959 BRIT, TEX.

Desmodium batocaulon A. Gray, 7632 BRIT, MEXU, TEX; 7639 ANSM, BRIT; 8268 TEX; (NMC 53469).

Desmodium cinerascens A. Gray, (TEX 221793).

Desmodium grahamii A. Gray, 8383, 8889.

Desmodium neomexicanum A. Gray, 6071; 6085 BRIT, MEXU, TEX; 6089, 6092; 7700 ANSM, BRIT; 8551; (NMC 48795; TEX 221226).

Desmodium retinens Schltdl., 8730 TEX.

Eysenhardtia parvifolia Brandegee *

Eysenhardtia schizocalyx Pennell *

Eysenhardtia spinosa Engelm. ex A. Gray, 4655; 5441 BRIT; 5452, 8622 BRIT; (NMC 46780, 46820).

Galactia macreii M. A. Curtis, 7861; 8499a TEX. 
Galactia wrightii A. Gray, 2663 ANSM, BRIT; 4642 MEXU; 4660 ANSM, MEXU; 4805; 4862 ANSM, BRIT; 4967; 7861 BRIT, TEX; 8175 ANSM; 8224 MEXU; 8658 MEXU; 8677 BRIT, MEXU.

Hosackia repens G. Don, (TEX 180398).

Indigofera miniata Ortega, (TEX 220662).

Indigofera ornithopodioides Schltdl. \& Cham., 5235 ANSM.

Indigofera sphaerocarpa A. Gray, 8237, 8595; (TEX 220715).

Lablab purpurea (L.) Sweet, 2950; 2951 NMC.

Lotus greenei (Wooton \& Standl.) Ottley ex Kearney \& Peebles, 7765 ANSM, BRIT, MEXU, TEX; (NMC 63650; TEX 180352).

Lotus oroboides (Kunth) Ottley ex Kearney \& Peebles, 2690 ANSM; 4152; 7246 TEX; 7378 BRIT; 8221 TEX; (TEX 180361, 180364, 180366).

Lotus plebeius (Brandegee) Barneby, 4299, 7145; 7638 TEX; 7682 TEX; 8299; 8379 TEX

Lupinus aff. delicatulus Sprague \& Riley, 8904.

Lupinus mexicanus Lag., 7271 MEXU; 7297 BRIT, MEXU, TEX; 7762 BRIT; 8440 ANSM, BRIT, MEXU; (TEX 180531).

Lupìnus sitgreavesii S. Watson, (TEX 180981).

Macroptilium gibossifolium (Ortega) A. Delgado, 5344, 5578; 7252 MEXU; 7681 BRIT; 7798 MEXU.

Marina calycosa (A. Gray) Barneby *

Medicago lupulina L., 2541.

Medicago sativa L., 2486, 4150, 6902 BRIT.

Melilotus indica (L.) All., 2483, 6898 BRIT, MEXU; 7116 BRIT; 9088.

Melilotus officinalis (L.) Lam., 4187.

Nissolia pringlei Rose 9008 TEX; (TEX 272784, 272785).

Nissolia wislizeni (A. Gray) A. Gray, 5274, 5288; 5309 ANSM, BRIT, MEXU, TEX; 5475 BRIT; 5516 BRIT; 5589 ANSM; 7241 ANSM, MEXU; 8164 BRIT; 8237 MEXU; (TEX 272796, 272804).

Pediomelum palmeri (Ockendon) Grimes, 4147.

Peteria scoparia A. Gray, 8034, 8135 BRIT, TEX; (TEX 272977, 272978, 272979).

Phaseolus acutifolius A. Gray var. acutifolius, 5858 BRIT, MEXU; (TEX 272987).

Phaseolus angustissimus A. Gray, 4646; 6435 MEXU, TEX.

Phaseolus coccineus L. var. formosus (Kunth) Maréchal, Mascherpa \& Stainier, (TEX 273068, 273079).

Phaseolus filiformis Benth., 8361 MEXU.

Phaseolus grayanus Wooton \& Standl., 7260 TEX; 7366 BRIT; 7713 TEX; 8598 TEX; 9145 MEXU. 
Phaseolus leptostachyus Benth., 9531 MEXU.

Phaseolus maculatus Scheele ssp. maculatus (citado como Phaseolus metcalfei Wooton \& Standl.); (TEX 273257).

Phaseolus maculatus Scheele ssp. ritensis (Jones) Freytag (citado como Phaseolus ritensis Jones) *.

Phaseolus parvulus Greene, 8901.

Phaseolus vulgaris L., 7108.

Rhynchosia macrocarpa Benth., 3435 MEXU; NMC 4645; 7663 MEXU.

Rhynchosia senna Gill ex Hook. var. angustifolia (A. Gray) Grear, 2472 BRIT; 4158, 5444

ANSM; 6430 BRIT; 6456 MEXU; 6868; 7011 BRIT; 7030 BRIT; 7079 BRIT.

Robinia pseudoacacia L., 7144 ANSM, BRIT.

Sophora gypsophila B.L. Turner \& Powell, 6954 ANSM, BRIT, MEXU, TEX; (TEX 274029, 274031).

Sophora nuttalliana B.L. Turner, (TEX 273954, 273955).

Tephrosia vicioides Schltdl., 4634.

Trifolium amabile Kunth var. amabile, 8521 BRIT.

Trifolium carolinianum Michx., 7281, 7341, 7350, 7380.

Trifolium mucronatum Willd. ssp. mucronatum, (TEX 274212, 274216).

Trifolium wormskioldii Lehm. var. ortegae (Greene) Barneby, 4297; 7348 BRIT; 7715

ANSM, BRIT; 8625.

Vicia leucophea Greene *

Vicia ludoviciana Nutt. var. ludoviciana, 8712 ANSM, BRIT; 8762; (TEX 274289).

Vicia pulchella Kunth, 8906 BRIT.

Wisteria sinensis (Sims) Sweet, 2760.

Zornia gemella (Willd.) Vogel, 7770.

Zornia reticulata Sm., 5299 BRIT, TEX; 5335 ANSM, NMC; 6129; 6136 BRIT; 8231a, 8683. Zornia thymifolia Kunth, (TEX 274479).

\section{LEGUMINOSAE (MIMOSOIDEAE)}

Acaciella angustissima (Mill.) Britton \& Rose var. angustissima, (TEX 152595, 152597). Acaciella angustissima var. chisosiana Isely, 5632 BRIT; 7815a ANSM, BRIT, TEX. Acaciella angustissima var. texensis (Nutt. ex Torr. \& A. Gray) L. Rico, 4952 ANSM, MEXU; 5342; 5869 BRIT; 6707, 7240; 7790 BRIT; 8160; 8595a, ANSM, MEXU; 8939 ANSM, BRIT.

Acacia berlandieri Benth., 9087 TEX.

Acacia biaciculata S. Watson, 8056 ANSM, BRIT, TEX; 8117 BRIT, NMC; (TEX 152800). 
Acacia constricta Benth., 4700; 4850 ANSM, BRIT; 4903 BRIT, MEXU; 8068; 8081 BRIT, MEXU; 8186 MEXU; 8475; 9063 BRIT.

Acacia farnesiana (L.) Willd., 2757.

Acacia glandulifera S. Watson, 6929 ANSM, BRIT; (TEX 46737, 153176, 153178).

Acacia greggii A. Gray var. arizonica A. Gray, 8936.

Acacia greggii A. Gray var. greggii, 2773 ANSM, BRIT, MEXU; 4857, 6932 ANSM, MEXU; 7042 MEXU; 7232 ANSM, TEX.

Acacia neovernicosa Isely, 5683 BRIT; 6871 MEXU, NMC; 7038 MEXU; 7180 BRIT; 8478

BRIT; 8486 TEX; 8695; 8700 MEXU; 8810 ANSM; 8959 TEX; 8994 MEXU; 8998; 9010 MEXU.

Acacia roemeriana Scheele, 2618; 6915 BRIT.

Acacia schaffneri (S. Watson) Herm. var. bravoensis Isely, 5241 ANSM, BRIT, MEXU; 7927.

Acacia wrightii Benth., 9085 TEX.

Calliandra eriophylla Benth. var. eriophylla, 2462 BRIT; 4625; 4949 BRIT; 6426 BRIT; 6756, 6926, 7808; 7809 BRIT; 7926 BRIT; 8474, 8692; 8800 MEXU; 8835 BRIT.

Calliandra humilis (Schltdl.) Benth. var. humilis, 7674.

Calliandra humilis Benth. var. reticulata (A. Gray) L. Benson, 7794 ANSM, BRIT; 8616; (NMC 52811; TEX 153964).

Desmanthus cooleyi (Eaton) Trel., 6768; 7016 BRIT; 7068 BRIT; 8046 ANSM, BRIT, MEXU; 8793 BRIT; 8796; (TEX 257274).

Desmanthus virgatus (L.) Willd., 4703.

Mimosa aculeaticarpa Ortega var. biuncifera (Benth.) Barneby, 2480; 6157 ANSM; 6419 BRIT, MEXU; 7143 ANSM, MEXU; 7178 BRIT, MEXU; 7613 BRIT, TEX; 8614 TEX; 8848 BRIT, TEX.

Mimosa dysocarpa Benth., 4622, 4654, 4861, 5022 ANSM; 5271; 5336 ANSM, TEX; 7242 MEXU; 8797 MEXU; (NMC 52860; TEX 230238, 230241).

Mimosa emoryana Benth. var. chihuahuana (Britton \& Rose) Barneby, 6755 BRIT; 6804 MEXU, TEX.

Mimosa emoryana Benth. var. emoryana, 5804 BRIT, 6921 BRIT; 8699.

Mimosa pringlei S. Watson var. pringlei, 4797 ANSM, MEXU; 4840 ANSM, MEXU; 8477;

8579 BRIT; 8181; 8798 MEXU; (NMC 52569; TEX 230533, 230534).

Mimosa texana (A. Gray) Small var. texana, 6865 ANSM, BRIT; 7040 BRIT, TEX.

Painteria elachistophylla (S. Watson) Britton \& Rose, 9086 ANSM, BRIT.

Painteria leptophylla (DC.) Britton \& Rose, 5451; 8300 BRIT; 8668 ANSM, BRIT.

Prosopis glandulosa Torr. var. glandulosa, (TEX 96275). 
Prosopis glandulosa Torr. var. torreyana (L. Benson) M.C. Johnst., 5142; 5164 MEXU; 5716 ANSM; 6924 MEXU; 7051 BRIT; 8806 MEXU; (TEX 96302, 96312, 96340).

Prosopis laevigata (Kunth ex Willd.) M.C. Johnst., 6732.

Prosopis velutina Wooton, 4793 ANSM, BRIT, MEXU; 5651 BRIT, MEXU; (TEX 96534). Zapoteca media (M. Martens \& Galeotti) H.M. Hern., 6140 TEX.

\section{LINACEAE}

Linum aristatum Engelm. *

Linum puberulum (Engelm.) Heller, (NMC 4890; TEX 254705).

Linum rupestre (A. Gray) Engelm. \& A. Gray, (TEX 254721, 254754).

Linum vernale Wooton, 7699 ANSM.

\section{LOASACEAE}

Cevallia sinuata Lag., 5105; 5157 ANSM; 5169, 5172, 5814, 7192.

Eucnide bartonioides Zucc., (TEX 181054).

Eucnide sp., 6423 ANSM, TEX.

Mentzelia asperula Wooton \& Standl., 7984 ANSM; 8018 ANSM, TEX; TEX 253461).

Mentzelia hispida Willd., (TEX 212111, 212115).

Mentzelia incisa Urb. \& Gilg., 7624 TEX.

Mentzelia lindheimeri Urb. \& Gilg, 8170 ANSM, TEX.

Mentzelia mexicana H.J. Thomps. \& Zavort., 6966 TEX; (TEX 181346).

Mentzelia multiflora (Nutt.) A. Gray, 2612 ANSM; 8150 ANSM, BRIT, TEX; (TEX 181363, 181364, 181365).

Mentzelia saxicola H.J. Thomps. \& Zavort., (TEX 181384, 181389, 181393).

Mentzelia texana Urb. \& Gilg, 5509 ANSM; 8472.

\section{LOGANIACEAE}

Buddleia marrubiifolia Benth., 5779, 6930; 8704 ANSM; 9015.

Buddleia scordioides Kunth, 5165; 5697 ANSM, BRIT.

\section{LYTHRACEAE}

Cuphea wrightii A. Gray, 9389.

Lythrum californicum Torr. \& A. Gray, 8636 ANSM, BRIT.

\section{MALPIGHIACEAE}

Aspicarpa hirtella L. C. Rich., 5021, 5030; 5036 ANSM, NMC; 7685 ANSM; 8435; 5036; 5545 BRIT. 
Aspicarpa humilis (Benth.) Juss., 4635, 4671; 4866, 5021 TEX; 5545; 9048 BRIT.

Aspicarpa hyssopifolia A. Gray, 7651 ANSM; 8204; 8235 ANSM.

Janusia gracilis A. Gray, 7805 ANSM, BRIT, NMC; 7824, 9077.

\section{MALVACEAE}

Abutilon fruticosum Guill. \& Perr., (TEX 140401).

Abutilon incanum (Link) Sweet, 7863 TEX; 8791 BRIT; 9072 ANSM, BRIT, TEX.

Abutilon malacum S. Watson, 9082 ANSM, BRIT, TEX; (TEX 140534).

Abutilon mollicomum (Willd.) Sweet, (TEX 140568).

Abutilon wrightii A. Gray, 9734.

Anoda crenatiflora Ortega, (TEX 140847).

Anoda cristata (L.) Schltdl., 6065, 6078, 6083, 7153, 8125, 8188; 8387 BRIT; (TEX 140915, 140920).

Anoda lanceolata Hook. \& Arn. *

Anoda pentaschista A. Gray, 5853 TEX.

Anoda thurberi A. Gray, (TEX 74131).

Gossypium hirsutum L., 7904 BRIT.

Herissantia crispa (L.) Brizicky, 4647 ANSM; 4664, 5453 TEX; 7810.

Hibiscus acicularis Standl. *

Hibiscus coulteri A. Gray 9060; (TEX 74859, 74862).

Hibiscus denudatus Benth., 7816 BRIT; 7877 BRIT; (TEX 74885, 74887, 74890).

Malva parviflora L., 6903 ANSM, BRIT, TEX.

Malvastrum coromendelianum (L.) Garcke, 2482 ANSM.

Malvella lepidota (A. Gray) Fryxell, 6350 ANSM.

Malvella leprosa (Ortega) Krapov., 7230 ANSM, BRIT, TEX; (TEX 73670).

Malvella sagittifolia (A. Gray) Fryxell, (TEX 73680).

Meximalva filipes (A. Gray) Fryxell, 6941 ANSM, TEX.

Rhynchosida physocalyx (A. Gray) Fryxell, 5467, 7089; 7172 BRIT.

Sida abutifolia Mill., 2633, 5040 TEX; 5228; 5316 NMC; 5435; 6477 BRIT; 7102; 7818; 8159

ANSM, 8943; (NMC 52841, 52862; TEX 72178, 72206).

Sida aff. lindheimieri Engelm. \& A. Gray, 2632.

Sida procumbens Sw. (NMC 48793).

Sphaeralcea angustifolia (Cav.) G. Don var. angustifolia, 2766, 5201; 5230 TEX; 7074; 8923

ANSM.

Sphaeralcea angustifolia (Cav.) G. Don var. cuspidata A. Gray, 2767; 6918 ANSM; 8971

TEX.

Sphaeralcea coccinea (Pursh) Rydb., 8158 BRIT; 8864 ANSM, BRIT, TEX. 
Sphaeralcea hastulata A. Gray, (TEX 72927).

Sphaeralcea incana Torr. ex A. Gray, (TEX 72937).

\section{MARTYNIACEAE}

Proboscidea louisianica (Mill.) Thell. ssp. fragrans (Lindl.) Bretting, 4639, 7866; (TEX 105116, 105125).

Proboscidea parviflora (Wooton) Wooton \& Standl., 7933 ANSM, TEX; (TEX 105149).

\section{NYCTAGINACEAE}

Acleisanthes acutifolia Standl., (TEX 191666, 191667).

Acleisanthes chenopodioides (A. Gray) R.A. Levin, 4684; 6434 ANSM; 7179 BRIT; 8115 ANSM; (TEX 95105, 95017).

Acleisanthes lanceolata (Wooton) R.A. Levin var. maloneana (B.L. Turner) B.L. Turner, (TEX 95897, 95902).

Acleisanthes longiflora L., 5331 ANSM, NMC; 5573; 7090 ANSM; 7135 BRIT; (TEX 49909, 49912, 49915).

Acleisanthes megaphylla (B.A. Fowler \& B.L. Turner) B.L. Turner, (TEX 46143, 95903, 95904, 95905).

Allionia choisyi Standl., 6371, 6374; 7918 ANSM, BRIT, TEX.

Allionia incarnata L., 5108; 5112 BRIT; 5203 ANSM; 5229, 6823; 7091; 7842 BRIT, 7879 BRIT; 8078; 8977 BRIT; 8996; 9010; (NMC 43883; 46801).

Boerhavia coccinea Mill., 5217; 5220 ANSM; 5493, 5796; 7052; 7213 ANSM, BRIT; 7187 ANSM; (TEX 95130).

Boerhavia gracillima Heimerl, 5762 TEX; 6869 ANSM, TEX.

Boerhavia intermedia M.E. Jones, 4839; 7804 ANSM, BRIT; (NMC 46871, 71429; TEX 95169).

Boerhavia linearifolia A. Gray, 7905 BRIT, TEX.

Boerhavia spicata Choisy, 4619, 4800; 4839 NMC; 5101; 5135 TEX; 5795 NMC; 5873; 7856

BRIT, TEX; 8047 ANSM; 8169 BRIT.

Commicarpus scandens Standl., 7854 BRIT, TEX.

Cyphomeris gypsophiloides (M. Martens \& Galeotti) Standl., 8804a; (TEX 95243).

Mirabilis albida (Walt.) Heimerl, 6813 ANSM, TEX; 7591 ANSM, BRIT, TEX; NMC; 7607.

Mirabilis hirsuta (Pursh) MacMill., 8240 NMC.

Mirabilis linearis (Pursh) Heimerl, 5597; 7014 ANSM, BRIT; (NMC 52819).

Mirabilis longiflora L., 5573 ANSM, BRIT; 7315 ANSM, BRIT; (NMC 51818).

Mirabilis oblongifolia (A. Gray) Heimerl, (TEX 95324, 95326). 
Mirabilis polonii Le Duc, 4300.

Nyctaginia capitata Choisy, 5703 ANSM; 5715 BRIT; 8061, 8091 BRIT.

\section{OLEACEAE}

Forestiera angustifolia Torr., 9011 TEX; (TEX 156644, 156661).

Forestiera neomexicana A. Gray, (TEX 182690).

Fraxinus americana L., 7035 BRIT, TEX.

Fraxinus greggii A. Gray, (TEX 156848, 156873, 156896).

Fraxinus papillosa Lingelsh., 8911 ANSM, BRIT, TEX.

Fraxinus velutina Torr., 7243 ANSM, BRIT; 8403 BRIT; (TEX 156993, 156994, 156995).

Menodora scabra A. Gray var. laevis (Wooton \& Standl.) Steyerm., 5628 TEX; 5631 ANSM, BRIT; 9067.

Menodora scabra A. Gray var. ramosissima Steyerm., 6791 BRIT; 7107 ANSM; 7820, 8488 ANSM, BRIT, TEX; 9009 ANSM, TEX.

\section{ONAGRACEAE}

Calylophus hartwegii (Benth.) P.H. Raven ssp. filifolius (Eastw.) Towner \& P.H. Raven, 7185 TEX; 8155 ANSM.

Calylophus hartwegii (Benth.) P.H. Raven ssp. hartwegii, 2473; 4169 ANSM; 4686, 6765, 7071; 7112 ANSM, BRIT; 7925 BRIT; (TEX 160015, 160017, 160018, 160024).

Calylophus toumeyi (Small) Towner, 7134 ANSM, TEX; (NMC 44823, 48779; TEX 160103).

Calylophus tubicola (A. Gray) P. H. Raven var. tubicola, (TEX 160126).

Epilobium ciliatum Raf., 7342 ANSM, BRIT; 7721 ANSM, TEX.

Gaura boquillensis P.H. Raven \& D.P. Gregory, (NMC 48780; TEX 160621).

Gaura coccinea (Nutt.) Pursh var. arizonica Munz, 7007 ANSM.

Gaura coccinea Nutt. var. epilobioides (Kunth) Munz, 4151 ANSM; 7080 ANSM, BRIT; 7277 ANSM, BRIT, TEX; 7737 ANSM, TEX; 8372 ANSM; 8404 BRIT.

Gaura coccinea (Nutt.) Pursh var. parviflora (Torr.) F.C. Gates, 8165 ANSM, BIRT; (TEX 160659).

Gaura hexandra Ortega var. gracilis (Wooton \& Standl.) P. H. Raven \& D.P. Gregory, 5524 ANSM; 5480 TEX.

Gaura macrocarpa Rothr. *

Ludwigia palustris (L. ) Elliot*

Ludwigia peploides (Kunth) P.H. Raven ssp. peploides, 2610 ANSM; 4295; 5463 BRIT; 7154 ANSM; (TEX 161232).

Oenothera albicaulis Nutt., (TEX 161280). 
Oenothera brachycarpa A. Gray, (TEX 161291, 161292).

Oenothera kunthiana (Spach) Munz, (TEX 161359).

Oenothera laciniata Hill ssp. pubescens (Willd. ex Spreng.) Munz, 7726 TEX; 8892 TEX.

Oenothera macrosceles A. Gray, (TEX 161408).

Oenothera pallida Lindl. ssp. runcinata (Engelm.) Munz \& W.M. Klein, 7298a ANSM, TEX; 7315a.

Oenothera pringlei (Munz) Munz, 4156.

Oenothera rosea Aiton, 6906 BRIT; 6992 ANSM; 7165; 7265 BRIT; (NMC 67350).

Oenothera speciosa Nutt. *

\section{OXALIDACEAE}

Oxalis alpina (Rose) Kunth, 7375 ANSM.

Oxalis caerulea (Small) Kunth, 7792 ANSM.

Oxalis corniculata L. var. pilosa (Nutt. ex Torr. \& A. Gray) B.L. Turner, 4161, 6790; 7067 ANSM; 7160a ANSM; 7278 BRIT, MEXU, NMC; 8133B; (TEX 254095).

Oxalis corniculata L. var. wrightii (A. Gray) B.L. Turner, 7160 ANSM; 7754 ANSM; (TEX 254182).

Oxalis drummondii A. Gray *

Oxalis latifolia Kunth, 7114 ANSM, BRIT; 7606 ANSM; 8028 BRIT; (NMC 52568; TEX 254346).

\section{PAPAVERACEAE}

Argemone albiflora Hornem., (TEX 191780).

Argemone mexicana L. ssp. mexicana, 7036 ANSM, BRIT, TEX; (TEX 46188).

Argemone turnerae G.W. Ownbey var. turnerae, (TEX 46189, 147445, 147446, 147447; 147451).

Eschscholtzia mexicana Greene, 2754 ANSM.

\section{PHYTOLACCACEAE}

Rivina humilis L., 7852 ANSM, BRIT.

\section{PLANTAGINACEAE}

Plantago australis Lam. ssp. hirtella (Kunth) Rahn, (TEX 89516).

Plantago elongata Pursh, 5644 BRIT, TEX.

Plantago eriopoda Torr., (TEX 89587, 89588).

Plantago hookeriana Fisch. \& C.A. Mey. *

Plantago aff. lanceolata L., 7161 ANSM, TEX. 
Plantago linearis Kunth var. mexicana (Link) Pilger, 7365 ANSM; 7611 BRIT, TEX; 8875 BRIT.

Plantago linearis Kunth var. villosa Pilger, 7290 TEX.

Plantago major L., 7714 ANSM, BRIT, TEX.

Plantago ovata Forssk. *

Plantago patagonica Jacq., 4180.

Plantago rhodosperma Decne., 7158 ANSM, TEX.

PLUMBAGINACEAE

Plumbago scandens L., 4661, 9124; (TEX 228428).

\section{POLEMONIACEAE}

Gilia incisa Benth. *

Gilia mexicana A. Grant \& V.E. Grant*

Gilia rigidula Benth. ssp. acerosa (A. Gray) Wherry, 7559 ANSM; (TEX 192435).

Gilia rigidula Benth. ssp. rigidula, 4607 ANSM; 7037 ANSM, BRIT.

Gilia stewartii I. M. Johnst., 9656; (TEX 192666, 192669, 192673).

Ipomopsis aggregata (Pursh) V.E. Grant ssp. formosissima Wherry, (TEX 46870, 46871, 46874).

Ipomopsis havardii (A. Gray) V.E. Grant, (TEX 192515).

Ipomopsis longiflora (Torr.) V.E. Grant ssp. neomexicana Wilken, (TEX 46903).

Ipomopsis pinnata (Cav.) V.E. Grant, 8321 ANSM; 8365 ANSM; 8505 ANSM, BRIT, TEX. Ipomopsis macombii (Torr.) V.E. Grant, 8377 ANSM; (TEX 213003).

Ipomopsis pringlei (A. Gray) W.C. Martin \& C.R. Hutchins, (TEX 213046, 213049).

Phlox nana Nutt., 7150 ANSM, BRIT, TEX; 7282 TEX; 7370 ANSM.

Loeselia coerulea (Cav.) G. Don, 5790 ANSM, BRIT, TEX; 6641 ANSM.

\section{POLYGALACEAE}

Monnina wrightii A. Gray, 8734 BRIT; 8770.

Polygala alba Nutt., 4166, 4608, 5026, 5069, 8451 BRIT; 9457; (TEX 233951).

Polygala hemipterocarpa A. Gray, (TEX 222140).

Polygala lindheimeri A. Gray var. parvifolia Wheelock, 7182 ANSM; (TEX 222265).

Polygala macradenia A. Gray var. macradenia, 6917 ANSM, BRIT; (TEX 222374, 222287).

Polygala nudata Brendegee, (TEX 222449, 222459, 222474).

Polygala obscura Benth. var. obscura, 5430 TEX; 5432; 7675 BRIT; 7930 TEX; 8014; 8076 ANSM; 8183; (NMC 52809). 
Polygala scoparioides Chodat, 6927 TEX; 6983 ANSM; (TEX 187663, 200468, 200495).

Polygala semialata S. Watson, (TEX 187685).

Polygala watsonii Chodat, (TEX 188909).

\section{POLYGONACEAE}

Eriogonum abertianum Torr., 8980 ANSM; 9068; (TEX 109738, 109770).

Eriogonum atrorubens Engelm. var. atrorubens, (TEX 109788, 109798).

Eriogonum inflatum Torr. \& Frém., 4947 ANSM, TEX; 7276 ANSM, BRIT; 7730 ANSM; 7795 ANSM; 8244 BRIT.

Eriogonum jamesii var. undulatum (Benth.) S. Stokes, (TEX 109945, 109947, 109987, 172009).

Eriogonum polycladon Benth., 7174 ANSM, TEX.

Eriogonum tenellum Nutt., (NMC 48777; TEX 172073, 172074, 172077, 172079, 172095).

Eriogonum wrightii Torr. ex Benth., 4685 ANSM; 6702 ANSM.

Polygonum glabrum Willd., 7741 TEX.

Polygonum pensylvanicum L., 7684 BRIT; 7720 ANSM, BRIT; 7730; 8217 ANSM, TEX; 8244.

Polygonum punctatum Raf., 5798 ANSM, BRIT, TEX.

Rumex crispus L., 7264 TEX; 7287 ANSM, TEX; 7309 TEX; (TEX 172538).

Rumex mexicanus Meisn., 8269 TEX; 5706 ANSM.

Rumex triangulivalvis (Danser) Rech., (TEX 172589).

\section{PORTULACACEAE}

Portulaca oleracea L., 4847, 4864 ANSM; 5455; 7853 TEX.

Portulaca pilosa L., 5367; 5414 BRIT; 5424, 5375, 5547, 8035, 8156; 8666 ANSM; 9025.

Portulaca suffrutescens Engelm., 7833 BRIT, TEX.

Talinum angustissimum (A. Gray) Wooton \& Standl., 5371, 5863; 8127 ANSM; 8132 ANSM, BRIT.

Talinum aurantiacum Engelm., 5863 ANSM; 5868; 6837a; 7831 ANSM, BRIT; 8971 BRIT; 9437.

Talinum paniculatum (Jacq.) Gaertn., 4665, 5872; 5571 ANSM, BRIT; 7640 ANSM; 7830 TEX; 8009 ANSM, BRIT.

\section{PRIMULACEAE}

Anagallis arvensis L. *

Samolus ebracteatus Kunth var. cuneatus Small, 7916 ANSM, BRIT; (TEX 228361, 228286, 228287). 


\section{PYROLACEAE}

Monotropa hypopitys L., 8728.

\section{RANUNCULACEAE}

Clematis drummondi Torr. \& A. Gray, 5682 TEX; 8012 TEX; 8095 ANSM, BRIT, TEX; 8133; (NMC 71578, 72597; TEX 240119, 240124, 240127).

Delphinium subscandens Ewan, (TEX 240336, 240338).

Delphinium tenuisectum Greene, (TEX 240343).

Delphinium wislizeni Engelm., 8502 ANSM, BRIT, TEX; 8902 ANSM, BRIT, NMC.

Ranunculus cymbalaria Pursh *

Ranunculus forreri Greene 7748 ANSM, BRIT, TEX.

Ranunculus hydrocharoides A. Gray, 7156 ANSM, BRIT; 7329 TEX; 7716 ANSM, BRIT, TEX.

Ranunculus petiolaris DC. var. arsenei (L.D. Benson) T. Duncan, (TEX 240643).

Thalictrum grandifolium S. Watson, 7269 BRIT; 7597 ANSM; 7687 BRIT; 7706 BRIT, TEX; 9144.

Thalictrum pinnatum S. Watson, 7269 BRIT; 8005 BRIT.

\section{RESEDACEAE}

Oligomeris linifolia (Vahl) J.F. Macbr., 9118.

Reseda luteola L., 2753 ANSM.

\section{RHAMNACEAE}

Adolphia infesta (Kunth) Meisn., 6840 ANSM, BRIT, TEX; (TEX 264717, 264718, 264719).

Ceanothus fendleri A. Gray var. venosus Trel., 9306c; (TEX 265034).

Ceanothus greggii A. Gray, 8013 TEX; (TEX 265131).

Condalia correllii M.C. Johnst., (TEX 263595).

Condalia ericoides (A. Gray) M.C. Johnst., 6959 ANSM, BRIT; 7176 ANSM, BRIT; 8162; (TEX 263602).

Condalia fasciculata I.M. Johnst., (TEX 263628).

Condalia warnockii M.C. Johnst. var. warnockii, 5657 ANSM; 7909 ANSM, TEX; 8802; (TEX 263508, 263511, 263516).

Ziziphus obtusifolia (Hook. ex Torr \& A. Gray) A. Gray var. obtusifolia, 7234 ANSM.

\section{ROSACEAE}

Fallugia paradoxa (D. Don) Endl. ex Torr., 5496, 5501 NMC; 5506; 7225 ANSM; 7251 ANSM, BRIT; 8722 ANSM, BRIT; (TEX 150888, 150893, 150896). 
Potentilla thurberi A. Gray, 8774; 9147; (TEX 151419).

Prunus serotina Ehrh. var. rufula (Wooton \& Standl.) McVaugh, (TEX 151654).

Prunus serotina Ehrh. var. serotina, 8031 ANSM, TEX.

Prunus serotina Ehrh. var. virens (Wooton \& Standl.) McVaugh, 8745 TEX.

Purshia ericaefolia (Torr. ex A. Gray) Henr., 8496 ANSM, BRIT.

Purshia mexicana (D. Don) Henr., 2463 ANSM.

Rubus neomexicanus A. Gray *

\section{RUBIACEAE}

Bouvardia multiflora (Cav.) Schult. \& Schult., (TEX 178220).

Bouvardia ternifolia (Cav.) Schltdl., 5079 ANSM; 7258 ANSM; 7976 ANSM; (TEX 178323, 178324).

Cephalanthus occidentalis L, 2606.

Diodia teres Walter, 5450 TEX; 5608 TEX; 6159 ANSM; 8206; (NMC 48773; TEX 42233, 42236).

Galium mexicanum Kunth ssp. asperulum (A. Gray) Dempster, 7736; 8359 ANSM; 8461 BRIT; 8915a ANSM, BRIT; (TEX 42472).

Galium mexicanum Kunth ssp. mexicanum, (TEX 42481).

Hedyotis intricata Fosberg, (TEX 42587, 45286, 45291).

Hedyotis nigricans (Lam.) Fosberg var. nigricans, (TEX 45381).

Hedyotis nigricans (Lam.) Fosberg var. rigidiuscula (A. Gray) Shinners, 7379 ANSM; 7647 ANSM, BRIT.

Hedyotis rubra (Cav.) A. Gray, 7998 TEX; (TEX 45508).

Hedyotis wrightii (A. Gray) Fosberg, 6894 ANSM; 7305 BRIT; 7317 ANSM, BRIT, TEX; (TEX 45541, 45543).

Relbunium microphyllum (DC.) Hemsl., 8007 ANSM; (TEX 42500, 42502, 42504).

Richardia tricocca (Torr. \& A. Gray) Standl., 6808 ANSM, TEX.

Richardia sp. 7385 TEX.

Spermacoce hirta L., 5586 ANSM; 6160, 8214; 8516 ANSM, TEX.

\section{RUTACEAE}

Ptelea trifoliata L. ssp. angustifolia (Benth.) V. L. Bailey, 8002a ANSM, BRIT.

Thamnosma texanum (A. Gray) Torr., 6970 BRIT; 7136; (NMC 47326).

\section{SALICACEAE}

Populus deltoides Marshall, 7992 ANSM, BRIT, TEX.

Populus fremontii S. Watson, (TEX 244186, 244188). 
Populus tremuloides Michx., 8895 ANSM; (TEX 244232).

Salix bonplandiana Kunth, (TEX 171015, 171053).

Salix gooddingii C. R. Ball var. gooddingii, 5461 ANSM, TEX; (TEX 171085).

Salix lasiolepis Benth., (TEX 171155).

\section{SAPINDACEAE}

Cardiospermum dissectum Radlk., 8282 ANSM, BRIT; (TEX 213356, 231755).

Cardiospermum halicacabum L., 2604; 5263 ANSM; 8416 BRIT; 8556 BRIT; 8868.

Sapindus saponaria L. var. drummondii (Hook. \& Arn.) L.D. Benson, 7046 BRIT; 7229 ANSM, BRIT, NMC.

Ungnadia speciosa Endl., 7960 ANSM, BRIT.

\section{SAURURACEAE}

Anemopsis californica (Nutt.) Hook. \& Arn., 5636 BRIT; 5648 ANSM; 7913 BRIT.

\section{SAXIFRAGACEAE}

Heuchera rubescens Torr. var. versicolor Greene, 8767 ANSM, BRIT, NMC.

\section{SCROPHULARIACEAE}

Agalinis peduncularis (Benth.) Pennell, 8322 ANSM, TEX.

Buchnera obliqua Benth., 8442 BRIT.

Castilleja chromosa A. Nels., 7733 ANSM; 8905 ANSM, TEX.

Castilleja aff. elongata Penn., 4977, 6012.

Castilleja integra A. Gray, 4934; (TEX 179455, 179456).

Castilleja lanata A. Gray, 5079, 7976.

Castilleja mexicana A. Gray, 5446 ANSM; 7788 ANSM, BRIT, TEX; 8289 ANSM; (NMC 52102, 52817).

Castilleja nervata Eastw., 7286 ANSM, BRIT, TEX; 7320 TEX; 8440 BRIT.

Castilleja patriotica Fernald, 8503 ANSM, TEX; (TEX 179705).

Castilleja rigida Eastw., (TEX 179722, 179723).

Leucophyllum frutescens (Berland.) I. M. Johnst., 6848a.

Leucophyllum laevigatum Standl., (TEX 106092).

Leucophyllum minus A. Gray, (TEX 106160, 106171).

Maurandya antirrhiniflora Willd. ssp. antirrhiniflora, 2613, 8001; (TEX 106402).

Maurandya wislizeni A. Gray *

Mecardonia vandellioides (Kunth) Pennell * 
Mimulus glabratus Kunth, 2603 ANSM; 7157 ANSM, BRIT, TEX; 7349 ANSM, TEX; (TEX 106660).

Mimulus guttatus DC., 6989 BRIT, TEX; (TEX 106711, 106717).

Penstemon ambiguus Torr., (TEX 106853).

Penstemon apateticus Straw, 7171.

Penstemon barbatus (Cav.) Roth var. torreyi (Benth.) A. Gray, 7169 ANSM; 7273 BRIT, TEX; (TEX 106944).

Penstemon campanulatus (Cav.) Willd. ssp. chihuahuensis Straw, 7373 BRIT, TEX; 7603

BRIT; 7701 ANSM; 8305 BRIT; 8378 ANSM; (TEX 107067).

Penstemon dasyphyllus A. Gray, 4991 ANSM; 8182 ANSM; (TEX 107171).

Penstemon havardii A. Gray, 5050 BRIT, TEX; (TEX 253598).

Penstemon stenophyllus A. Gray, 8533 BRIT; (TEX 107494).

Schistophragma intermedia (A. Gray) Pennell *

Seymeria bippinnatisecta Seem., 7702 ANSM, TEX.

Seymeria decurva Benth. ex DC., 8356 ANSM, TEX.

Veronica polita R. E. Fr., 6887a ANSM, BRIT, TEX; 8733 TEX.

\section{SIMAROUBACEAE}

Castela stewartii (C. H. Müll.) Moran \& Felger, 6876 ANSM, BRIT; 9005 BRIT.

\section{SOLANACEAE}

Chamaesaracha conioides (Moric. ex Dunal) Britton, 6734 ANSM, BRIT, TEX; 6981 BRIT; 6956 ANSM, BRIT; 8096, 8938 ANSM, BRIT, TEX.

Chamaesaracha coronopus (Dunal) A. Gray, 5155, 5876; 6947 ANSM; 7897 BRIT, 8142 ANSM; (TEX 226576, 226579).

Datura quercifolia Kunth, 5498 ANSM; 5511 BRIT.

Datura wrightii Regel, 4892 BRIT; 7940 ANSM; 7652 ANSM, TEX; 8173.

Lycium berlandieri Dunal var. berlandieri, 5687 ANSM, TEX; (TEX 227157, 227165).

Lycium puberulum A. Gray, 9002 TEX; (TEX 227407, 227408, 227413).

Nicotiana glauca Graham, 4872 BRIT.

Nicotiana obtusifolia M. Martens \& Galeotti, 4869 BRIT; 9084 ANSM.

Physalis caudella Standl., 8363 ANSM, TEX.

Physalis glutinosa Schltdl. var. eximia (Standl.) Waterfall, 4301.

Physalis hederaefolia A. Gray var. hederaefolia, 2662 ANSM; 7106 BRIT; 8893 ANSM,

BRIT; (TEX 227993, 227996, 227999).

Physalis hederaefolia A. Gray var. puberula A. Gray, 4658; 5596 BRIT.

Physalis leptophylla B. L. Rob. \& Greenm., 8573 ANSM, TEX. 
Physalis microphysa A. Gray, (TEX 219106).

Physalis philadelphica Lam., 7626 ANSM; (TEX 219189).

Physalis pringlei Greenm., (TEX 219217).

Physalis pubescens L. var. pubescens, 6747 ANSM, BRIT, TEX; 8190 ANSM.

Solanum americanum Mill., 5457 ANSM, BRIT; 7139 BRIT; 7971 BRIT.

Solanum brachystotrichum (Bitter) Rydb., 7744 ANSM.

Solanum citrullifolium A. Br., 5665 ANSM, BRIT; 5722; (TEX 219677, 219692).

Solanum douglasii Dunal, (TEX 219984).

Solanum elaeagnifolium Cav., 4174, 4801, 6942; 7245 ANSM.

Solanum heterodoxum Dunal, (TEX 218137, 218146).

Solanum rostratum L'Hér. ex Dunal, 5308, 5499, 5503; 7352 BRIT; 7767; (TEX 233147, 233152).

Solanum stenophyllidium Bitter, (TEX 219509).

Solanum aff. sisymbrifolium Lam.,6712 ANSM, TEX.

Solanum stoloniferum Schltdl., (TEX 187002, 218046, 218049).

Solanum tenuipes Bartlett var. latisectum Whalen, (TEX 233312, 233313, 233314).

Solanum tridynamum Dunal, (TEX 233382).

\section{STERCULIACEAE}

Ayenia microphylla A. Gray, (TEX 76884, 76909).

Ayenia pilosa Cristóbal, 4908 BRIT; 4999; 5032 ANSM; 7848; 7868 ANSM, BRIT, TEX.

Ayenia pusilla L., 9069 TEX; (TEX 76814).

\section{TAMARICACEAE}

Tamarix aphylla (L.) H. Karst., (TEX 159106).

Tamarix ramosissima Ledeb., 5679 BRIT; (TEX 159116).

\section{ULMACEAE}

Celtis laevigata Willd. var. reticulata (Torr.) L. Benson, 6466 ANSM; 8860 BRIT.

Celtis pallida Torr., 4841 BRIT; 8987; 9001.

\section{URTICACEAE}

Parietaria pensylvanica Muhl. ex Willd. *

Urtica gracilenta Greene, 7999 TEX.

\section{VALERIANACEAE}

Valeriana sorbifolia Kunth var. sorbifolia, 8727 ANSM, BRIT, NMC. 


\section{VERBENACEAE}

Aloysia gratissima (Gillies ex Hook.) Troncoso, 4826, 5133, 5156; 5380 BRIT; 7828 ANSM; (NMC 66778; TEX 99122).

Aloysia wrightii (A. Gray) Heller, 5867, 6723; 6849 ANSM; 7039; 7985 TEX; 8297 BRIT; 8557; 8809 NMC; (TEX 99043).

Bouchea longiflora (Moldenke) G. L. Nesom, (TEX 99284, 99285).

Bouchea prismatica Kuntze var. brevisrostra Grenzeb., 4624 ANSM; 5034 ANSM, TEX; 5113; 5233 ANSM; (TEX 99318).

Bouchea spathulata Torr. *

Citharexylon brachyanthum (A. Gray) A. Gray *

Glandularia bipinnatifida (Nutt.) Nutt. var. ciliata (Benth.) B.L. Turner, 5292 BRIT; 6264 TEX; 6440 TEX; 6558 TEX; 6606 TEX; 6764 BRIT; 6934 BRIT, TEX; 7010; 7082 TEX; 7132 BRIT, TEX; 7142 TEX; 7218 TEX; 7476 TEX; 7797 ANSM; 7896; 8941 BRIT, TEX; (TEX 98092, 99968).

Glandularia bipinnatifida (Nutt.) Nutt. var. latilobata (L.M. Perry) B.L. Turner, (TEX 98084, 98085, 98086, 98088).

Glandularia chiricahensis Umber, (TEX 98082, 98087).

Glandularia elegans (Kunth) Umber var. asperata Perry, 6421; 7210.

Lantana camara L. *

Lantana macropoda Torr., 4618; 5810 TEX, BRIT; 7043 ANSM, BRIT, TEX; 7817 ANSM, BRIT, TEX; 9080 TEX.

Lippia curtisiana Moldenke*

Lippia graveolens Kunth, (TEX 100466, 100472).

Lippia incisa (Small) E. D. Schulz, 6454 BRIT; 6462; 6471.

Phyla nodiflora (L.) Greene, (TEX 100802, 100803).

Priva aspera Kunth, (TEX 100874).

Priva grandiflora (Ortega) Moldenke, (TEX 100923, 100926).

Priva mexicana (L.) Pers., 7620 ANSM, BRIT, TEX; (TEX 101010).

Tetraclea coulteri A. Gray var. angustifolia (Wooton \& Standl.) A. Nelson \& J. F. Macbr., 6796, 7086; 7939 ANSM; 8112 ANSM.

Verbena canescens Kunth, 5519, 5521 ANSM, TEX; (NMC 48782; TEX 101310).

Verbena cloverae Moldenke, (TEX 101497).

Verbena gracilis Desf., 7058 ANSM; 7757 ANSM, BRIT; 8397 ANSM, BRIT; (TEX 101517).

Verbena neomexicana (A. Gray) Small var. hirtella Perry 5811 BRIT; 6740; 6766 BRIT; 6907 BRIT; 8805 ANSM, BRIT; 8984. 
Verbena neomexicana (A. Gray) Small var. neomexicana, 6740 TEX; 7128 TEX; 5466; (BRIT, MEXU, NMC, NY 8131; TEX 200849, 200850).

Verbena scabra Vahl *

VIOLACEAE

Hybanthus verticillatus (Ortega) Bail. var. verticillatus, 4181 ANSM; 7063 BRIT; 7226.

Viola sp. (material sin flor ni fruto), 9700.

VISCACEAE

Arceuthobium vaginatum (Kunth) Eichler *

Phoradendron bolleanum (Seem.) Eichler, (TEX 108190).

Phoradendron capitellatum Torrey ex Trel. *

Phoradendron coryae Trel. 8512 ANSM, BRIT, TEX; (NMC 52823).

Phoradendron juniperinum Engelm. *

Phoradendron tomentosum (DC.) Oliver, 6985 ANSM, BRIT, TEX.

\section{VITACEAE}

Parthenocissus quinquefolia (L.) Planch., 8891.

Vitis arizonica Engelm., 6987 ANSM, BRIT; 7362 ANSM, BRIT, TEX; 8761 BRIT; (TEX 263157).

\section{ZYGOPHYLLACEAE}

Kallstroemia grandiflora Torr., (TEX 259209, 259219).

Kallstroemia hirsutissima Vahl, 4796 TEX.

Kallstroemia parviflora Norton, 4965; 5418 ANSM; 8087; (TEX 259296).

Larrea tridentata (DC.) Coville, 6925, 8487, 8958.

Tribulus terrestris L., 5491, 7101, 7827. 